\title{
Overlapping neural processes for stopping and economic choice in orbitofrontal cortex
}

\author{
Pragathi Priyadharsini Balasubramani ${ }^{1}$ \& Benjamin Y. Hayden ${ }^{2}$
}

1. School of Medicine and Dentistry, University of Rochester Medical Center, Rochester, NY. 14627

2. Neuroscience and Center for Magnetic Resonance Research, University of Minnesota

Minneapolis, MN. 55455

Corresponding Author:

Pragathi Priyadharsini Balasubramani 430, Elmwood Ave, Box 278917

University of Rochester Rochester, NY, USA, 14627

Email: pragathipriyadharsini@gmail.com

Acknowledgements: This work was supported by an R01 (DA038615) to BYH. We thank Meghan C. Pesce for help with data collection. 


\section{ABSTRACT}

36 Economic choice and stopping are not traditionally treated as related phenomena.

37 However, we were motivated by foraging models of economic choice to hypothesize that

38 they may reflect similar neural processes occurring in overlapping brain circuits. We

39 recorded neuronal activity in orbitofrontal cortex (OFC), while macaques performed a

40 stop signal task interleaved with a structurally matched economic choice task. Decoding

41 analyses show that OFC ensembles predict successful versus failed stopping both before

42 the trial and immediately after the stop signal, even after controlling for value

43 predictions. These responses indicate that OFC contributes both proactively and

44 reactively to stopping. Moreover, OFC neurons' engagement in one task positively

45 predicted their engagement in the other. Finally, firing patterns that distinguished low

46 from high value offers in the economic task distinguished failed and successful trials in

47 the stopping task. These results endorse the idea that economic choice and inhibition may

48 be subject to theoretical unification. 


\section{INTRODUCTION}

50 Stopping (sometimes referred to as inhibition) and economic choice are two major

51 brain functions that have historically been studied independently. Nonetheless, there is

52 some reason to think that they may spring from shared processes. For example, several

53 psychiatric conditions, including depression and addiction, impair both processes, and

54 greater impairment of both is associated with greater disease progression (Iacono et al.,

55 2008; Nestler et al., 2002; Volkow et al., 2011). Second, both are closely associated with,

56 and empirically linked to, the broader concept of self-control (Berkman et al., 2016;

57 Hayden, 2018; Inzlicht et al., 2014; Shenhav, 2017). Third, both tend to activate a similar

58 set of brain regions, including the pre-motor cortex, the ventrolateral prefrontal cortex,

59 basal ganglia, and the thalamus (Aron, 2007; Aron and Poldrack, 2006; Cisek, 2012;

60 Cisek and Kalaska, 2010; Hampshire and Sharp, 2015; Sakagami and Pan, 2007; Schall

61 et al., 2002).

62 The idea that stopping and choice may have a deeper relationship is motivated by

63 certain foraging-inspired approaches to economic choice (Cisek, 2012; Cisek and Pastor-

64 Bernier, 2014; Hayden, 2018; Hayden and Moreno-Bote, 2018; Kacelnik et al., 2011;

65 Krajbich et al., 2010; Stephens and Krebs, 1986). A core tenet of these approaches is that

66 the brain's decision-making systems are evolved to make accept-reject decisions

67 (Kacelnik et al., 2011; Ojeda et al., 2018; Pirrone et al., 2017; Shapiro et al., 2008;

68 Vasconcelos et al., 2010). Even ostensibly binary economic choices, in this view, reflect

69 a pair of (potentially interacting) accept-reject choices. Each accept-reject decision, in

70 turn, involves choosing whether to pursue an option or refrain from pursuit. Accepting

71 involves selecting the attended or activated option, or, more abstractly, performing the 
72 afforded action (Cisek and Kalaska, 2010; Cisek and Pastor-Bernier, 2014; Hayden and

73 Moreno-Bote, 2018). Rejecting involves countermanding the afforded action. A classic

74 binary economic choice, then, may be seen as two related decisions about whether to go

75 or stop choosing the attended option or the afforded action (Hayden, 2018).

76 This way of looking at choice is consistent with some recent studies that suggest

77 that binary choice involves a serial, not parallel, consideration of options (Krajbich et al.,

78 2010; Rich and Wallis, 2016; Strait et al., 2014; reviewed in Hayden and Moreno-Bote,

79 2018). These studies and others indicate that attention is largely limited to a single option,

80 which is evaluated, often relative to the other one (Lim et al., 2011; Rich et al., 2017;

81 Rudebeck and Murray, 2014; Strait et al., 2014 and 2015; Xie et al., 2018). Choice, then,

82 presumably occurs relative to a single option that is evaluated relative to a background

83 value, which includes the value of choosing the other option (Shapiro et al., 2008;

84 Vasconcelos et al., 2010). However, this work does not directly tie economic choice and

85 reward processing to stopping processes.

86 Here we sought to test the overlap hypothesis by comparing neuronal activity in

87 an economic choice task with that observed in a stopping task. We focused on the

88 orbitofrontal cortex (OFC). The centrality of OFC in economic choice is largely

89 undisputed, although its specific role remains to be determined (Padoa-Schioppa, 2011;

90 Rich et al., 2017; Rudebeck and Murray, 2014; Schoenbaum et al., 2009; Wallis, 2007;

91 Wilson et al., 2014). It is clear, nonetheless, that activity of Area 13 of OFC correlates

92 with the values of offers and of chosen options, and is likely to be critical for value

93 comparison as well (Padoa-Schioppa, 2013; Padoa-Schioppa and Assad, 2006;

94 Raghuraman and Padoa-Schioppa, 2014). In contrast to its clear role in choice, the 
95 contribution of the OFC to stopping remains disputed. On one hand, a good deal of work

96 argues against a direct inhibitory role for the OFC (Chudasama et al., 2006; Ghods-

97 Sharifi et al., 2008; Rudebeck and Murray, 2014; Schoenbaum et al., 2003; Stalnaker et

98 al., 2015). However, multiple studies give the OFC at least some role in inhibition

99 (Bryden and Roesch, 2015; Chikazoe et al., 2009; Dias et al., 1996; Eagle et al., 2007;

100 Horn et al., 2003; Majid et al., 2013; Mishkin, 1964; Roberts and Wallis, 2000). One

101 reason for the continued debate about the role of OFC in choice the lack of direct

102 evidence from the unit activity in this region in stopping tasks (but see: Bryden and

103 Roesch, 2015).

104 We hypothesized that OFC participates in both choice and stopping decisions in

105 similar ways, that is, by computing executive signals that promote (or fail to promote)

106 particular strategies. To test this hypothesis, we examined responses of OFC neural

107 populations recorded in two interleaved tasks, a stop signal task and an economic choice

108 task. The tasks were designed to have structures as similar as practically possible. We

109 were particularly interested in the questions of (1) whether and how the function of this

110 economic region includes stopping, and (2) whether neural response patterns related to

111 stopping correspond with patterns related to value. 


\section{RESULTS}

\section{Behavior in the stop signal task and economic choice task}

115 Subjects performed a standard stop signal task (similar but not identical to the

116 one used by Hanes and Schall (1995; Figures 1A and 1B and Methods). On each trial,

117 following a central fixation, subjects saw an eccentric target (go signal) that, if fixated,

118 provided a juice reward. On a subset of trials (33\%, called stop trials), a second signal

119 (stop signal) appeared at fixation and countermanded the previously instructed saccade.

120 Successful stopping trials were rewarded. Failed trials (trials in which a saccade was

121 made despite a stop signal) were not. Median reaction time to the target in go trials was

$1220.435 \mathrm{sec}$ and $0.272 \mathrm{sec}$ in subject $\mathrm{J}$ and subject $\mathrm{T}$, respectively (Figures 1C and 1D).

123 Average trial length including feedback time for subjects $\mathrm{J}$ and T were $3.66 \mathrm{~s}$ and $2.62 \mathrm{~s}$,

124 with the mean feedback start time as $1.78 \mathrm{~s}$ and $1.49 \mathrm{~s}$, respectively. Both subjects

125 showed typical behavior in this task; their performance in stop trials varied as a function

126 of time of presentation of stop signals relative to that of go signal (Figures 1E and 1F).

127 The delay between the go signal and the stop signal is called the stop signal delay

128 (SSD) and it varied randomly across trials. We estimated the SSD that leads to

129 approximately $50 \%$ successful stopping (SSD-50) because it can help in computing the

130 stop signal reaction time (SSRT, Logan, 1994; Logan and Cowan, 1984; Verbruggen and

131 Logan, 2008). The SSD-50 was $0.277 \mathrm{sec}$ for subject J and $0.131 \mathrm{sec}$ for subject T. SSRT

132 computed for SSD-50 was $0.158 \mathrm{sec}$ for subject $\mathrm{J}$, and $0.141 \mathrm{sec}$ for subject $\mathrm{T}$. These

133 values are typical of rhesus macaques in these tasks (e.g. Hanes and Schall, 1995; Ito et

134 al., 2003). 
135 We randomly interleaved stop signal trials with trials from an economic choice

136 task. This task was designed to have a similar structure to the stop signal task.

137 Specifically, forced choice trials were equivalent to go trials and choice trials were

138 equivalent to stop trials (see Methods for details). In the economic choice task, the offers

139 were associated with low (yellow), medium (blue), or high (magenta) reward value

140 (Figure 1B). The subjects chose either offer 1 (which appeared at the periphery, similar

141 to go signal) or, when it occurred, could choose the later-appearing offer 2 (which

142 appeared at the center, similar to stop signal). The delay for offer 2 was fixed and defined

143 by the measured stop signal delay computed from the stopping task. For simplicity, we

144 will use the terms accept and reject trials to refer to those in which the subject chose offer

1451 and offer 2 , respectively.

146 As anticipated, the two tasks showed similar behavior results. Median reaction

147 time in forced choice trials was $0.41 \mathrm{sec}$ and $0.27 \mathrm{sec}$ in subject $\mathrm{J}$ and subject $\mathrm{T}$,

148 respectively (Figures $\mathbf{1 G}$ and $\mathbf{1 H}$ ). The reaction time medians for choice trials in the

149 presence of offer 2 were lower that that in forced choice trials (Supplemental results-A).

150 On average, the total length of trials including the feedback time for subjects $\mathrm{J}$ and $\mathrm{T}$

151 were $3.86 \mathrm{~s}$ and $2.88 \mathrm{~s}$, with the mean feedback start time as $1.88 \mathrm{~s}$ and $1.70 \mathrm{~s}$,

152 respectively. Choice accuracy varied as a function of SSD in both subjects (Figures 1E

153 and 1F; Supplemental results-A) Both subjects showed similar behavioral effects in the

154 current trial of each task, as a function of previous trial conditions (Figures 1I and 1J for

155 subject J, Figures $1 \mathrm{~K}$ and $\mathbf{1 L}$ for subject T; refer to supplementary results $\mathbf{A}$ for

156 behavioral effects). 
B economic choice task paradigm fixation offer $1 \quad$ offer 2
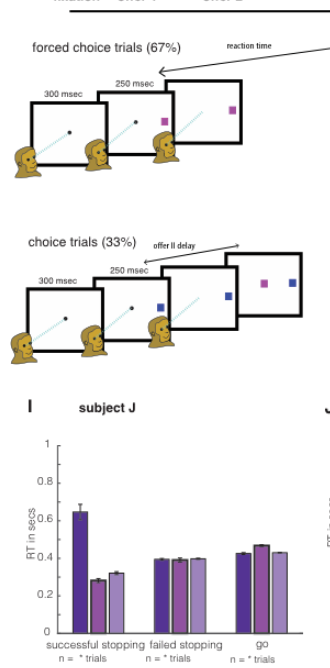
J shown).

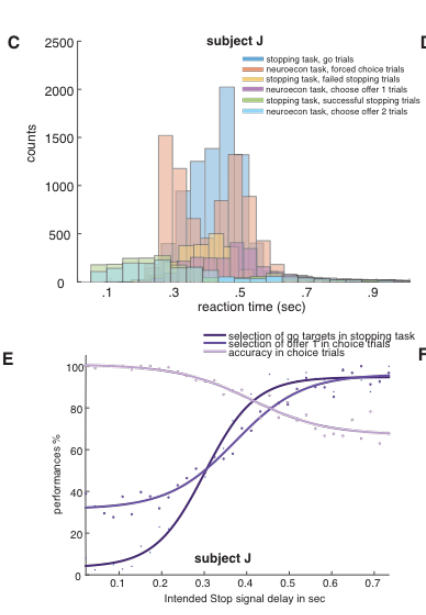

G
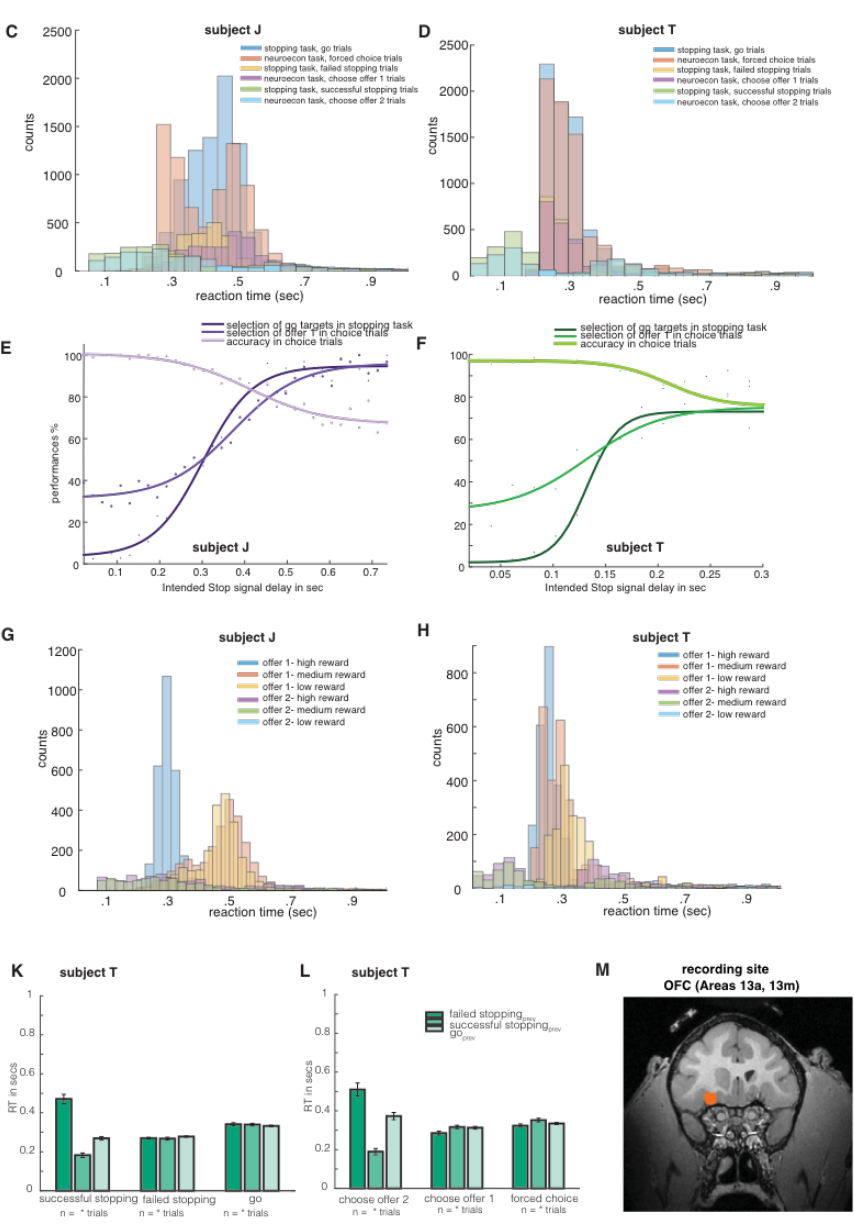
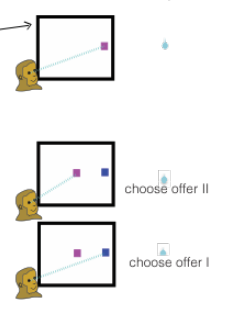

Figure 1. Task, anatomy, and subject behavior: task framework (A) stop signal task (B) economic choice task. Behavioral results for subject $J$ are presented in panels (C, E, G, I, J) and for subject T in (D, F, H, K, L) (C, D) reaction time distributions for various trial conditions of stop signal task (E, F) Inhibition function and accuracy of choices varied as a function of SSDs (G, H) reaction time distributions for various trial conditions of economic choice task. Previous trial had effects in reaction time behavior in $(\mathbf{I}, \mathbf{K})$ stop signal task and $(\mathrm{J}, \mathrm{L})$ economic choice task. Error bars represent SEM, and * denotes t-test significance with $p<0.05$. (M) recording site - Area 13 of OFC (scan from subject

\section{Overlapping sets of neurons participate in the two tasks}

We recorded responses of 96 neurons (52 in subject $\mathrm{J}$ and 44 in subject T) in Area

174 adjusted during recording based on analyses performed mid-experiment. Note that while 
175 this number is smaller than in some other studies, is it sufficient to detected the effects we

176 are interested in here.

177 We focused our analyses on four key time periods of the trial: (1) the $300 \mathrm{~ms}$

178 epoch before the trial started (pre-go signal epoch), which corresponds to fixation time

179 before the appearance of any stimulus targets; signal differences here presumably reflect

180 proactive control (Stuphorn and Emeric, 2012). (2) The variable time after the stop signal

181 and before the reaction time period (post-stop signal epoch). The post-stop epoch is

182 important because it is when inhibition generated in response to countermanding

183 commands would presumably occur, and has therefore been the focus of many studies of

184 stopping (Logan et al., 2015; Schall, 2001; Schall et al., 2002). It corresponds to the time

185 during which reactive control occurs (Stuphorn and Emeric, 2012). (3) The variable time

186 after the go signal and before the reaction time period (post-go signal epoch). The

187 equivalents of (2) and (3) in choice task are the variable times after offer 2, and offer 1,

188 respectively before the reaction time of a trial; these epochs denote the task related

189 activity in general; (4) the variable time between the beginning and end of feedback

190 reward (feedback epoch).

191 Neurons had diverse tuning profiles with near balance of sign. In stopping task,

$19244.79 \%$ of cells showed positive task tuning (response during task versus baseline), and

$19353.12 \%$ showed negative tuning, and the rest weren't modulated. In the economic choice

194 task, $45.83 \%$ of cells showed positive task tuning, and 51.04\% showed negative tuning,

195 and the rest weren't modulated. We examined the relationship between simple response

196 patterns in the two tasks on a cell-by-cell basis. We found similar neuronal activity when

197 comparing task related activity against baseline across tasks (which we call task-related 
198 tuning). Regression weights (task-related tuning coefficient) in one task positively

199 predicted the weights in the other task (Pearson correlation between tuning coefficients, $r$

$200=0.78, \mathrm{p}<0.001$, Figure 2A). (Note that these data come from separate sets of trials, so

201 there is no overlap in data used to estimate the two sets of tuning coefficients.) Moreover,

202 absolute response patterns (that is, unsigned regression weights) were also positively

203 correlated across the two tasks $(0.74, \mathrm{p}<0.001)$, indicating that it was the same set of

204 neurons involved in the two tasks, rather than distinct sets (for details on using this

205 method to interpret relationships between regression coefficients, see Blanchard et al.,

$2062015 a)$.

207 Next, we compared the coding of rewards in both tasks. For the stop signal task,

208 we looked at the differential coding of no-rewards during failed stopping versus rewards

209 in successful stopping; for the economic task, we looked at differential coding of varied

210 rewards associated with offers. Tuning coefficients for reward values were positively

211 correlated between both the tasks during the post-go signal period (between signed

212 coefficients, $r=0.3, p<0.001$, Figure $2 B$ ) and during the feedback epoch (between

213 signed coefficients, $\mathrm{r}=0.35, \mathrm{p}<0.001$, Figure $2 \mathrm{C}$ ). The positive correlation

214 demonstrates that the rewards are encoded in similar way across tasks. Moreover, the

215 unsigned correlation coeffcients were also correlated in both epochs $(r=0.27, p=0.01$

216 and $\mathrm{r}=0.28, \mathrm{p}=0.01$, respectively). This correlation indicates that coding of the two

217 types of value was handled by the same subset of neurons across the two tasks. 
A

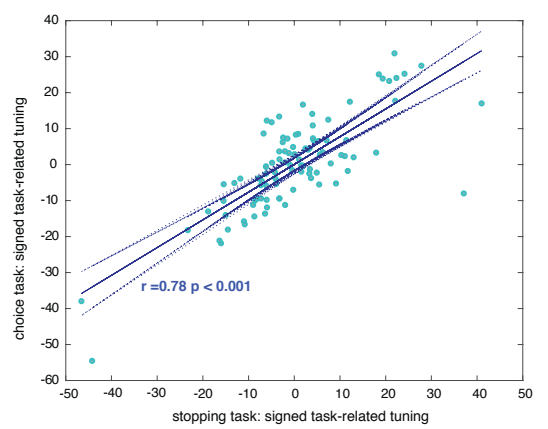

B

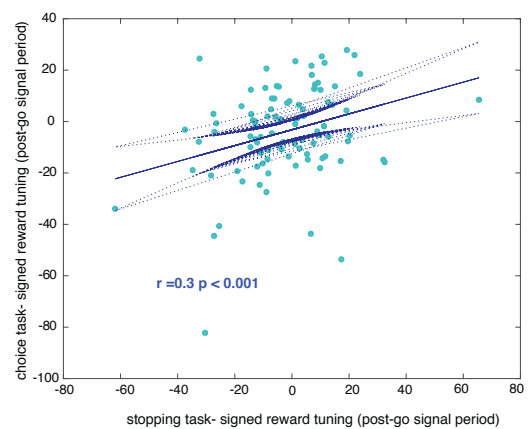

C

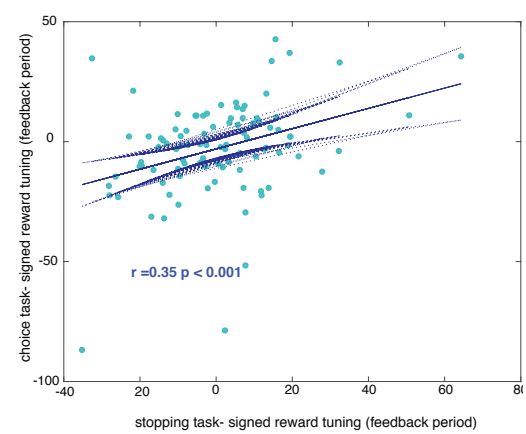

Figure 2- overlapping sets of neurons across stopping and choice tasks:

Correlations between signed $(\mathbf{A})$ task-related tuning coefficients, reward tuning coefficients in (B) post-go signal epoch (C) feedback epoch of stopping and economic choice tasks.

\section{Selectivity for stopping in single neurons}

The role of OFC in economic decisions is well established, but its role in stopping is not. Thus, a demonstration of functional overlap involves showing that it plays a role in stopping. Because of its relevance to the foraging hypothesis (see Introduction), we focused here on the determination of successful from failed stopping. The two time periods of significant interest to this hypothesis include: 1) The post-stop signal period, and 2) the pre-go signal time period.

Responses of example neurons are illustrated in Figure 3. In neuron J19, firing rates following the go signal but before the SSRT were lower on successfully inhibited trials (1.8 spikes/sec) than on failed stopping trials ( 4.1 spikes/sec, Wilcoxon rank test, ranksum $=1480, p<0.05, n=567$ trials, Figure 3A). Note that there is a larger and more prominent modulation in firing rate later in the trial. Given its timing, this modulation likely relates to outcome monitoring and is too late to influence stopping. Another example neuron, T25, showed distinct patterns for successful and failed stopping trials even $500 \mathrm{msec}$ before the beginning of the trial (ranksum $=2080, \mathrm{p}<0.05, \mathrm{n}=579$ trials,

240 Figure 3B). 
The responses shown in Figure 3C and 3D are aligned to stop signal (time zero).

242 Figure 3C illustrates the activity of the same neuron shown in Figure 3A; its response

243 pattern showed significant differences between successful stopping trials (1.8 spikes /

$244 \mathrm{sec}$ ) and failed stopping trials (4.4 spikes / sec) that begin after the presentation of stop

245 signal but before SSRT (ranksum = 1340, $\mathrm{p}<0.05$ ). Finally, neuron T10 (Figure 3D)

246 fired more vigorously on successful than on failed stopping trials at around $100 \mathrm{msec}$

247 after the SSRT (ranksum $=2229, \mathrm{p}<0.05$ ). The results also show that in OFC, the codes

248 for go and stop trials do not show simple and opposite activities for a saccade. This

249 contrasts with the activities of movement and fixation neurons in FEF, where inhibition is

250 driven by a rapid rise in firing rates of a specific subpopulation of neurons - fixation

251 neurons - that gate the activity of another subpopulation-movement neurons (Hanes and

252 Schall, 1995; Logan et al., 2015; Schall, 1991). Therefore, OFC neurons are rather

253 complex for a race model (Logan et al., 2015) to compute their stopping pattern.

254 To test for the possibility that our putative inhibition signals were just reward

255 correlates, we took advantage of trials collected from the economic choice task. The data

256 from this task allowed us to assess each neuron's tuning function for anticipated rewards.

257 Responses to different reward amounts by two example neurons are shown in Figures 3E

258 and 3F. We found tuning for anticipated reward values in the firing activity during the

259 reward feedback time period. For example, we observed a significant positive correlation

260 between reward amount and firing rate in neuron $\mathrm{J} 19(\mathrm{Q}=0.3138, \mathrm{p}<0.001$, Figure 3E),

261 this neuron shows similar reward related activity across tasks (Figure 3A, 3C). We also

262 show another neuron with a significant negative correlation to rewards (neuron T10, $\mathrm{Q}=$ -

$2630.143, p=0.04$, Figure 3F). Example individual neurons suggest the presence of diverse 
264 stopping and reward related neuronal codes at OFC. Furthermore, simple population

265 analyses don't inform about stopping patterns at OFC (refer to supplementary results-

266 B).
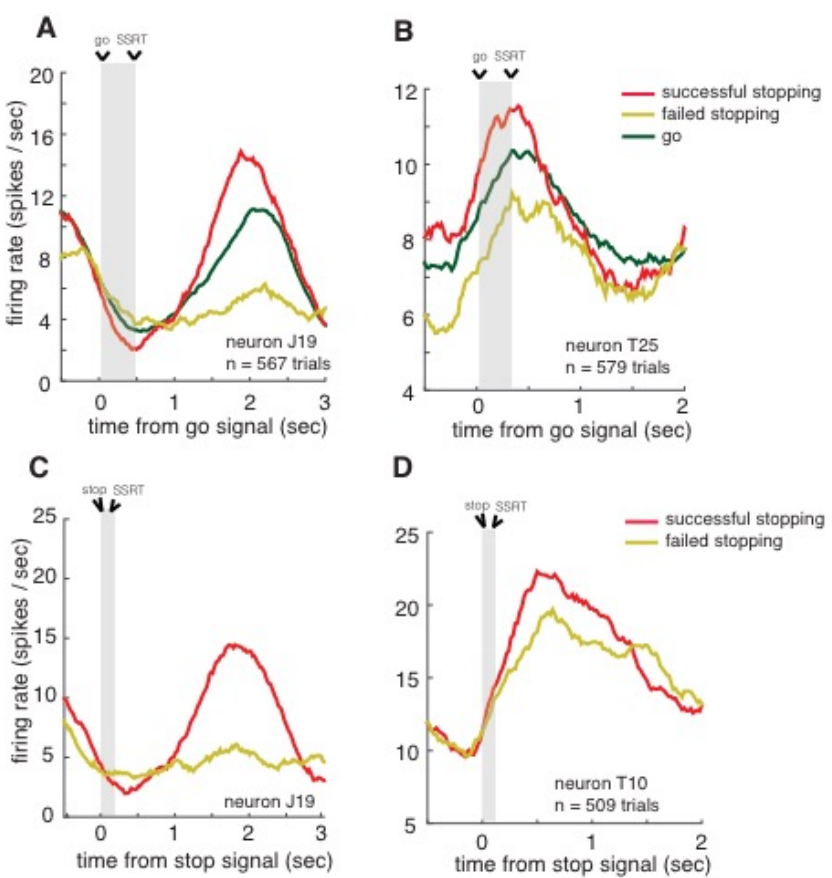

D
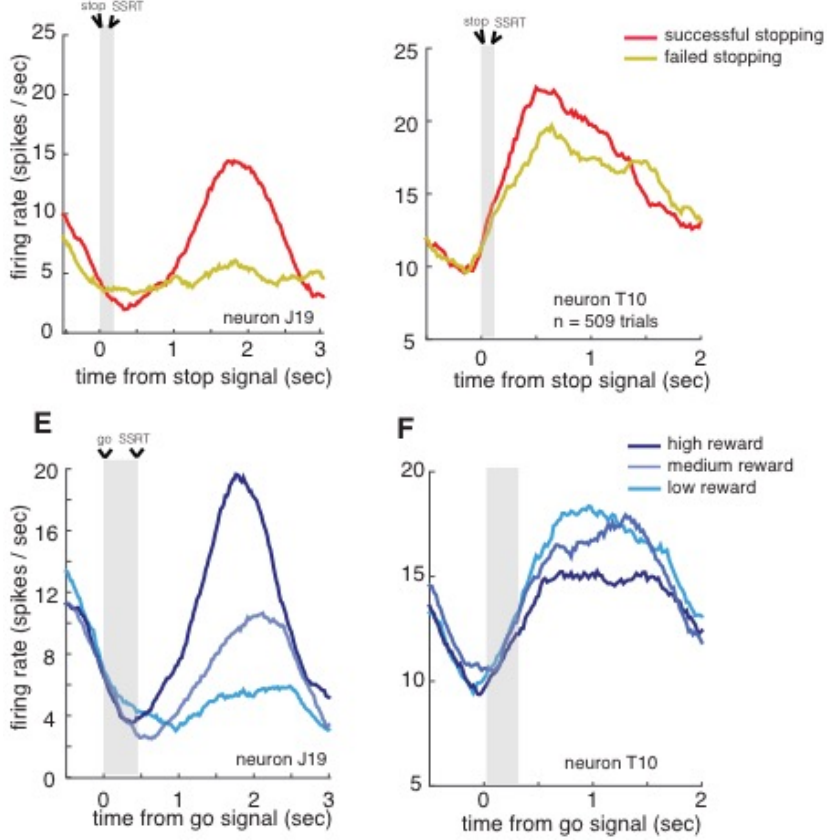

268 Figure 3- selectivity for stopping in sample neurons: Activity of example neurons during successful stopping, failed stopping and go trials are illustrated with respect to go signal in panels (A, B, E, F) and stop signal in (C, D) presentation time. Time from start of the go (stop) signal to SSRT is shaded all panels. Neuron in panel A shows significant difference in firing rates of successful and failed stopping trials before SSRT. Neuron in panel B shows difference even before the beginning of trial. Neuron in panel $\mathbf{C}$ is the same as panel $\mathbf{A}$, shows significant difference in firing rates of inhibition trials before stopping response time. Likewise, neuron in panel $\mathbf{D}$ shows difference around few msecs after SSRT. (E, F) Activity of example neurons in economic choice task: neuron in panel $\mathbf{E}$ is the same as panels $\mathbf{A}$ and $\mathbf{C}$. Its reward related activity after SSRT in the choice task parallels to that of stop signal task, and is positively 
280 correlated to the value. Neuron in panel F shows the opposite trend, and is 281 negatively correlated to the reward value.

Ensemble patterns distinguish successful from failed stopping

284 To compare whether similar ensemble patterns of activity predicted behavior in

285 the two tasks, we examined neural network decoders (classifiers). (Note that while we

286 used normalized data, see Methods, the normalization procedure did not alter our results,

287 see Supplementary results-C). We first trained decoders to analyze differences in

288 population activation patterns between successful and failed stopping in stop signal task;

289 We trained classifiers using two key epochs - post-stop signal epoch and pre-go signal

290 epoch. Testing of decoders for significant patterns differentiating successful and failed

291 stopping used $100 \mathrm{msec}$ moving boxcars stepping in $10 \mathrm{msec}$ intervals as input to the

292 classifier.

293 Because of the possibility of false positives, we were especially interested in

294 periods in which a decoder had several positive effects in adjacent bins (see Methods).

295 The post-stop signal decoder was able to classify success versus failure of inhibition

296 significantly in a series of 9 consecutive bins spanning 40 to $120 \mathrm{msec}$ after the stop

297 signal (these times indicate the starts of the 100 msec boxcars; see Methods on

298 procedures to determine statistical significance of a boxcar using chi-square statistics).

299 The corresponding numbers for individual subjects were 40 to $140 \mathrm{msec}$ in subject J and

30040 to $220 \mathrm{msec}$ in subject T (supplementary results-D). The post-stop signal pattern

301 series are unlikely to occur by chance $(\mathrm{p}<0.001$ in all cases, see Methods for specific

302 use of chi-square tests to quantify significance of consecutive bins). Since each bin spans

$303100 \mathrm{msec}$, the central point of the first element in this series provides a rough estimate of

304 the latency of the effect. That number was $\mathrm{t}=90 \mathrm{msec}$ for both subjects. 
306 subjects occurred before the stop signal reaction time of either subjects (the SSRTs were

$307140 \mathrm{msec}$ for subject J and $120 \mathrm{msec}$, also see supplementary results-D; Figure 4). We

308 call the central point the cancellation time; it measures the center point latency of first

309 statistically significant difference between successful and failed stopping trials for the

310 ensemble of neurons. The cancellation time is $90 \mathrm{msec}$ for both subjects. The cancellation

311 time preceded the average stopping response (SSRT) by $50 \mathrm{msec}$ in subject J, and by 30

312 msec in subject T, suggesting OFC's stopping-related patterns precede the stopping

313 response (see Discussion, and supplementary results-D).

314 We then examined the response differences of the pre-go signal decoder. We

315 observed a significant pattern difference between successful and failed stopping trials ( $p$

$316<0.001)$ in 36 number of boxcars, extending from 470 to $120 \mathrm{msec}$ before the go signal

317 (see Methods for details on chi-square statistics). For subject J, significant decoding was

318 observed in 35 number of boxcars during the time periods 460 to $120 \mathrm{msec}$; for subject T

319 it was 23 number of boxcars from 420 to $200 \mathrm{msec}$. These results indicate that the

320 upcoming success or failure of inhibition is decodable from OFC patterns even before the

321 start of the trial (Figure 4, also see supplementary results-D). The pre-go signal pattern

322 series are unlikely to occur by chance $(\mathrm{p}<0.001$ in all cases, see Methods for specific

323 use of chi-square tests to quantify significance of consecutive bins). Our results do not

324 tell us why this correlation exists, although one may infer that it reflects some internal

325 state that drives successful versus failed inhibition. Thus is it is a likely correlate of

326 proactive control. Overall, these results implicate Area 13 of OFC in the process of

327 regulating stopping decisions. 
348 values of pre-go and post-stop signal decoders (theoretical maximum), ' $r_{x \max }$ ' is $0.9058 \pm$

3490.0878 ; the value fell outside the central $98 \%$ of cross-validated data (and is significant at

Figure 4- ensemble analysis inform about stopping: Performance of (A) prego signal (B) post-stop decoders to distinguish successful versus failed stopping pattern. Insets in both panels illustrate sample projections of decoder's output responses ( $\mathrm{Y}$ - and Z-axes indicate the values of $\mathrm{O}_{1}$ and $\mathrm{O}_{2}$, for successful and failed stopping trials, respectively). Error bars indicate SEM, so non-overlap with the chance bar (horizontal dashed red line) is not sufficient to indicate statistical significance). Time points underneath through thick horizontal orange bars (and black tick marks) denote start time of $100 \mathrm{msec}$ boxcars having classification accuracy significantly above chance (i.e. 50\%) (chi-square test, p <0.05).

\section{The post-stop and pre-go decoders are statistically orthogonal}

We next examined how the pre-go and post-stop decoders related to each other.

We did so by comparing their weight vectors. We found a very low similarity between

them (Pearson correlation coefficient, $r=0.0008 \pm 0.0086$ ), suggesting the two epoch

patterns may be nearly statistically orthogonal and hence independent. A different

possibility is that this low correlation may be an artifact of noise. To test this second

possibility, we next performed a cross-validation procedure to estimate the maximum

range of measured cross-correlation values had the variables been fully correlated given

our noise properties. Cross correlation coefficient obtained between converged weight
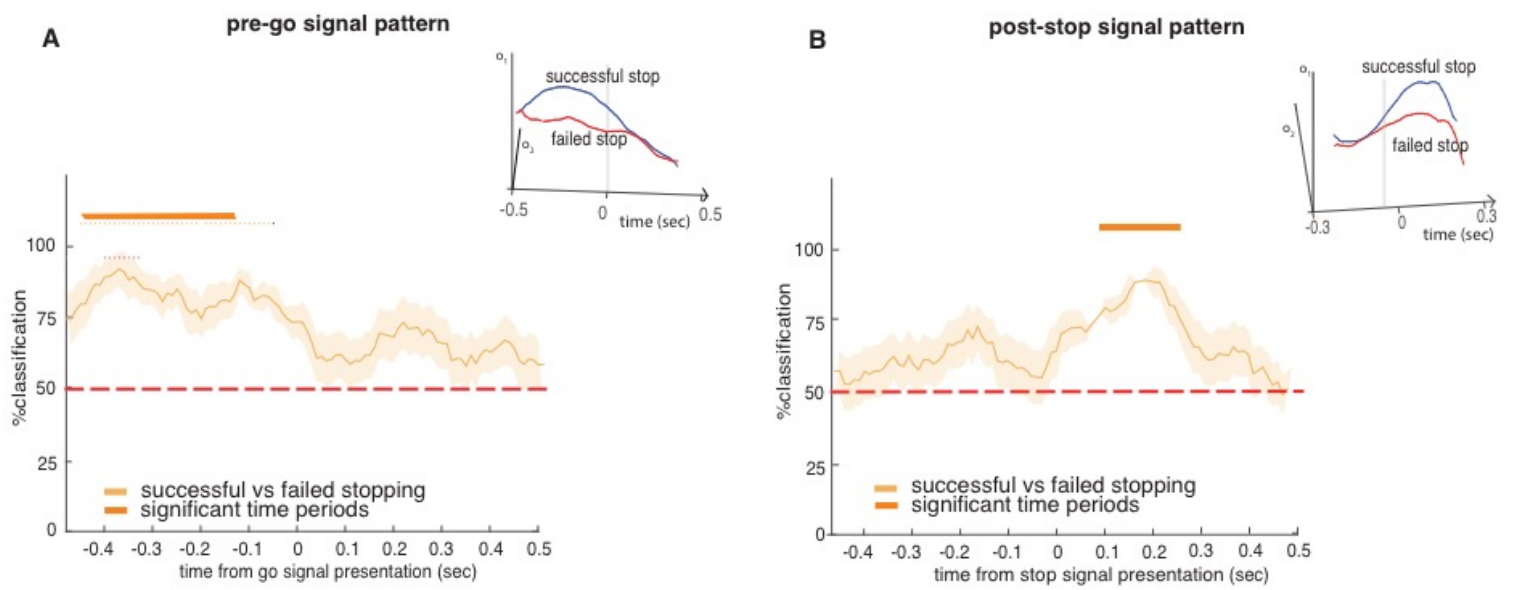

$350 \mathrm{p}<=0.01 ; 100$ randomizations, average of $r_{x-\max }$ from the randomized sets $=25.84 \pm$ 
351 0.82), substantiating statistical independency between pre-go and post-stop signal

352 decoder weight patterns.

353 Moreover, when the decoding performances for two decoders were compared,

354 they were significantly different during the time periods after the stop signal (t-stat $=$

355 6.0491, $\mathrm{p}=0.003)$. Similarly, they were significantly different even before the beginning

356 of trial ( $\mathrm{t}$-test, $\mathrm{t}$-stat $=8.8874, \mathrm{p}<0.001)$. The above results suggest that the two decoders

357 that predict successful versus failed inhibition are statistically orthogonal and thus

358 dissimilar. More broadly, these results suggest that the computations associated with

359 ostensible proactive and reactive control of stopping by OFC are distinct.

361 OFC encoding of stopping is not a by-product of value coding

362 The reward-encoding role of OFC is a hallmark of its function (Padoa-Schioppa,

363 2011; Rushworth et al., 2011; Schoenbaum et al., 2009; Schoenbaum et al., 2011;

364 Schultz, 2000; Wallis, 2007). We therefore wondered whether the stopping-related

365 activity that we observed might by an artifact of its reward roles. For example, it may be

366 that there is some undetectable natural variation in the relative subjective value of the

367 reward offered for correct performance. On trials in which the reward happened to have a

368 slightly lower value, the subject would be less motivated to perform correctly; this

369 fluctuation would then introduce a correlation between firing rates and successful

370 stopping (see, for example, Sugrue et al., 2005, for a similar argument about LIP

371 neurons).

372 If the stopping-related signals were a direct consequence of reward encoding for

373 every neuron, we would see a positive correlation between coding patterns for rewards 
374 and stopping. We computed a reward index for all neurons by regressing their responses

375 at feedback epoch against the outcomes themselves. We computed a stopping index for

376 all neurons by subtracting on their firing rate during successful and failed stopping before

377 SSRT (see Methods). We found no correlations between these indices in the post-stop

378 signal time period (Pearson correlation, $\varrho=0.09$, $p=0.4$, Figure 5). Nor did we find such

379 correlations in pre-go signal time period ( $\mathrm{Q}=-0.02, \mathrm{p}=0.82$, Figure 5).

380 This lack of correlation at the neural level may be a sign that the reward code and

381 the stopping code are different. It may also, in theory, be due to lack of sufficient data to

382 detect a significant effect. To test this idea, we performed a cross-validation analysis (See

383 Methods). Specifically, we reasoned that if insufficient data were the problem then a

384 within sample correlation would also produce no significant correlation. A positive

385 coefficient resulting from a within sample correlation, using randomly sampled half-sized

386 subsets, then, would indicate that our data have sufficient power to detect a significant

387 effect (Blanchard et al., 2015a). We thus tested whether the correlation coefficient for

388 stopping and reward indices fell below the bottom 5 percentile of the coefficients

389 obtained for within-group correlations. Indeed, the coefficient fell below 1st percentile of

390 that obtained for 100 randomizations in cross validation analysis. Figure 5 show no-

391 correlations between stopping and reward indices with $\mathrm{p}<=0.01$. 
$\begin{array}{ll}392 & \\ 393 & \text { Figure 5- un } \\ 394 & \text { stopping and } \\ 395 & \text { pre-go signal } \\ 396 & \\ 397 & \text { Overlapping fun }\end{array}$

$\begin{array}{ll}392 & \\ 393 & \text { Figure 5- unr } \\ 394 & \text { stopping and } \\ 395 & \text { pre-go signal } \\ 396 & \\ 397 & \text { Overlapping fun }\end{array}$

$\begin{array}{ll}392 & \\ 393 & \text { Figure 5- unr } \\ 394 & \text { stopping and } \\ 395 & \text { pre-go signal } \\ 396 & \\ 397 & \text { Overlapping fun }\end{array}$

$\begin{array}{ll}392 & \\ 393 & \text { Figure 5- unr } \\ 394 & \text { stopping and } \\ 395 & \text { pre-go signal } \\ 396 & \\ 397 & \text { Overlapping fun }\end{array}$

$\begin{array}{ll}392 & \\ 393 & \text { Figure 5- unr } \\ 394 & \text { stopping and } \\ 395 & \text { pre-go signal } \\ 396 & \\ 397 & \text { Overlapping fun }\end{array}$

$\begin{array}{ll}392 & \\ 393 & \text { Figure 5- unr } \\ 394 & \text { stopping and } \\ 395 & \text { pre-go signal } \\ 396 & \\ 397 & \text { Overlapping fun }\end{array}$

398

399

400

401

402

403

404

405

406

407

408

409

410

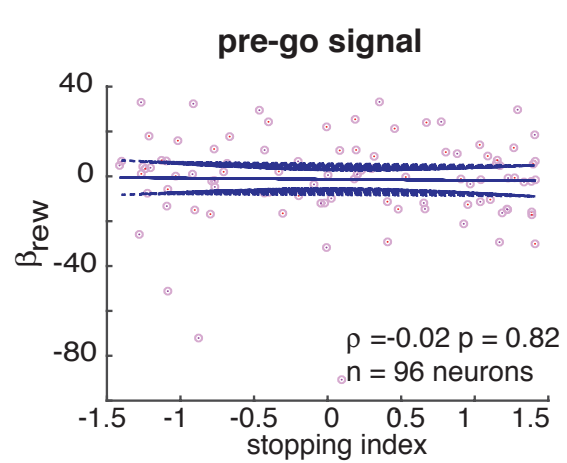
pre-go signal and (B) post-stop signal time period.
B

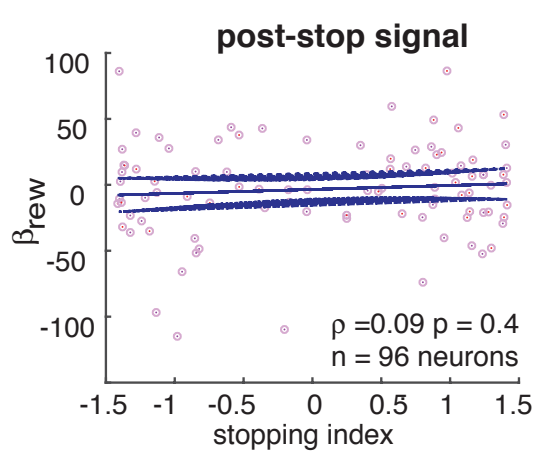

Figure 5- unrelated reward and stopping codes: Correlations between stopping and reward indices show no significant effect during $100 \mathrm{msec}$ in (A)

\section{Overlapping functional ensemble codes for stopping and for value}

We next examined the relationship between patterns that distinguished successful versus failed stopping of the stop signal task, and different reward values of the economic choice task. One potential confound in such an analysis is that OFC may encode action, and similar effects may reflect their shared actions (Feierstein et al., 2006; Grattan and Glimcher, 2014; Roesch et al., 2006; Yoo et al., 2018; Strait et al., 2016). To deal with this problem, for the analysis of economic choice task data, we used only trials in which the subject accepted the offer. Thus, action was the same - a saccade - in all cases.

411 stopping patterns showed significant similarities to time periods -140 to $-60 \mathrm{msec}, 420$ to

$412570 \mathrm{msec}$, from the presentation of offer 1 for patterns differentiating low versus high 
413 rewarding accept trials (Figure 6). We also trained a reversed-network with reversed

414 training and testing sets: trained with patterns distinguishing high versus low value-

415 accept trials, and tested with patterns distinguishing failed and successful stopping trials.

416 The significance of the reversed network, in contrast with the earlier, looks for the

417 presence of economic choice task related patterns in the stopping task trials. Similar to

418 the earlier, in reversed-network, training with patterns differentiating low versus high

419 rewarding accept trials of choice task, in the post-go signal epoch, showed significant

420 similarity to time periods around -230 to $-70 \mathrm{msec}, 420$ to $720 \mathrm{msec}$ from the presentation

421 of go signal for patterns differentiating failed versus successful stoppings, respectively

422 (Figure 6). This shows the presence of overlapping patterns between tasks, the neural

423 patterns relating to the decision process of one particular task-type can be tracked from

424 many time periods (pre trial onset and during the later parts of the trial) of the other task-

425 type.

426 Then, we trained with feedback epoch of successful versus failed stopping trials;

427 their patterns showed similarity to time periods 570 to $690 \mathrm{msec}$ after the presentation of

428 offer 1 of high versus low rewarding accept trials (Figure 6). In the reversed-network,

429 with characteristics described as the previous, training using high versus low rewarding

430 accept trials of choice task showed significant similarities to time periods around 190-220

431 msec and 400-440 msec of failed versus successful stopping patterns, respectively

432 (Figure 6). These results show the similarities of feedback related ensembles in both

433 tasks.

434 Reward promotes choice of the option it is associated with. The results show that

435 the patterns used to encode stopping in the stop signal task would correspond to patterns 
436 associated with value encoding in the economic choice task (Hayden, 2018). Particularly,

437 we found that task and feedback epoch related patterns of one task can be tracked from

438 the other, in time periods both before and during the trial, in a non-continuous and

439 dispersed manner. Similarities between neural patterns of both tasks may promote the

440 idea of shared strategies between stopping and choice decisions. We hypothesize that

441 reward related patterns could act as motivational forces when present in stopping

442 decisions. On the other hand, presence of stopping related patterns in choice decisions

443 could facilitate internal action strategies guiding the choice.

A

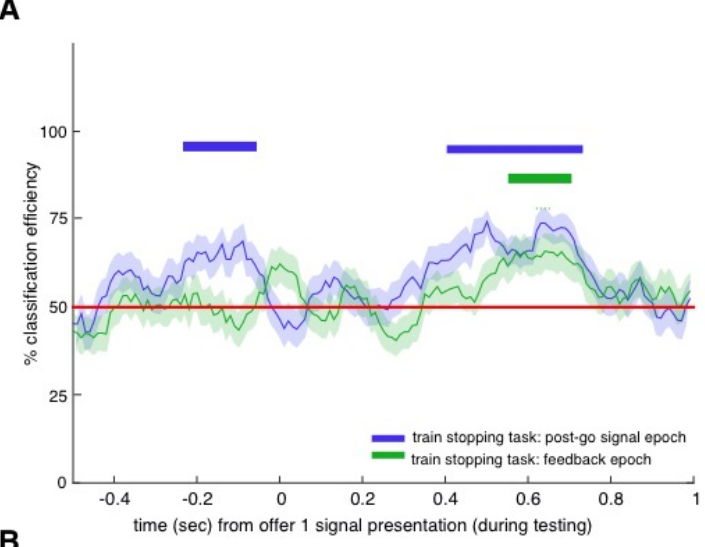

B

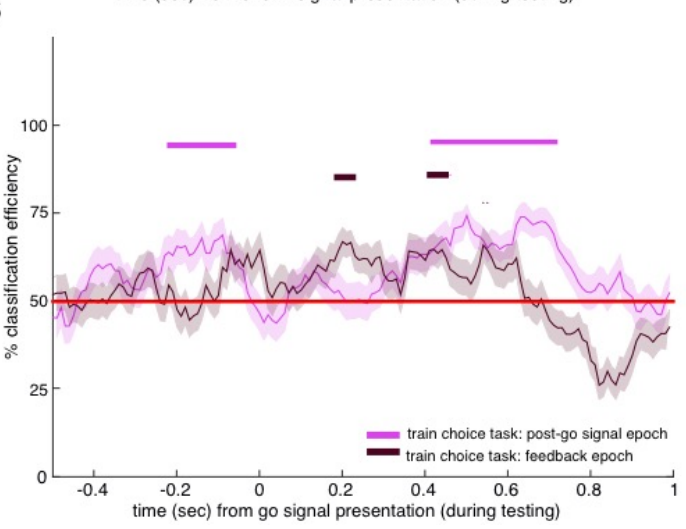

446 Figure 6- overlapping functional ensemble codes for stopping and choice:

447 Performance of decoders trained on (A) stopping task (B) choice task on post448 stop signal epoch (blue, magenta) and feedback (green, brown) epochs. Error 449 bars represent SEM. Time points underneath the thick horizontal highlighted bars 450 (blue, green, magenta, brown) of each result, as labelled in the legend, denote 451 the start time of $100 \mathrm{msec}$ boxcars having percent accuracies of classification 
bioRxiv preprint doi: https://doi.org/10.1101/304709; this version posted April 20, 2018. The copyright holder for this preprint (which was not certified by peer review) is the author/funder. All rights reserved. No reuse allowed without permission.

452 above chance of $50 \%$ (chi-square test, $p<0.05$ ). Red line indicates chance $453(50 \%)$ performance for both decoders. 


\section{DISCUSSION}

455 We examined responses of neurons in Area 13 of the OFC in two tasks, one an

456 implementation of a classic stopping task and the other a simple economic choice task

457 with similar structure. Although the economic role of this region in choice is well

458 established, its role in stopping is not. Our finding that OFC ensembles predict stopping

459 both before the trial and immediately before the stopping response demonstrate that this

460 region does participate in regulation of stopping. The timing of the two stopping-related

461 patterns is reminiscent of the times associated with reactive and proactive control,

462 respectively (Braver, 2012; Braver et al., 2007; Chen et al., 2010; Chikazoe et al., 2009;

463 Hanes et al., 1998; Ito et al., 2003; Majid et al., 2013; Stuphorn et al., 2000; Stuphorn et

464 al., 2010; Stuphorn and Emeric, 2012). Moreover, by interleaving the tasks, we were able

465 to show that it is largely the same neurons participating in both processes. Finally, we

466 show that the patterns that differentiate value in the choice task can distinguish failed

467 from successful stopping in the stop signal task. These results thus support the hypothesis

468 that stopping and economic choice reflect common computations occurring in

469 overlapping circuits.

470 These results provide evidence in favor of the hypothesis that the neural

471 processes that regulate stopping relate to the ones that regulate economic choices. In

472 foraging theory, decisions are generally framed as accept-reject (Blanchard and Hayden,

473 2015; Kacelnik et al., 2011; Stephens and Anderson, 2001; Stephens and Krebs, 1986).

474 From this perspective, binary choices, the mainstay of behavioral economics and

475 microeconomics, are better thought of as two somewhat independent accept-reject

476 decisions (Hayden and Moreno-Bote, 2018; Kacelnik et al., 2011). Each accept-reject 
477 decision, in turn, functions like its classic foraging counterpart, that is, as a choice

478 between pursuing and refraining from pursuit (Stephens and Krebs, 1986; Freidin et al.,

479 2009; Kacelnik et al., 2011). In other words, what appears to be a binary choice may

480 actually be a pair of countermanding stopping decisions (Hayden, 2018). Our results

481 provide tentative neural evidence in support of this idea.

482 These results also invite a reconsideration of the role of the OFC. This region,

483 especially Area 13, is sometimes cast as a specialist in economic functions (Padoa-

484 Schioppa, 2011; Wallis, 2007). Our results challenge that narrow view and endorse a

485 broader view that encompasses stopping as well. We doubt that the role of OFC is limited

486 to these two functions. Its other functions likely include contingent (rule-based)

487 decisions, working memory, switching, and conflict monitoring (Bryden and Roesch,

488 2015; Lara et al., 2009; Mansouri et al., 2014; Meyer and Bucci, 2016; Sleezer et al.,

489 2016; Sleezer et al., 2017). All these functions, including stopping and economic choice,

490 can arguably be placed within the larger category of executive functions. Like stopping,

491 executive functions more broadly are generally associated with dorsal prefrontal

492 structures (Miller, 2000; Miller and Cohen, 2001). Some of these functions may also be 493 part of the repertoire of the OFC as well.

494 Stopping is often associated with dorsal prefrontal structures, such as FEF, and

495 with subcortical structures, like SC (Hanes and Schall, 1995; Logan et al., 2015; Schall,

496 1991). Our work suggests that the role of OFC is qualitatively different than these

497 regions. Specifically, we find evidence that single neurons were neither consistently

498 associated with a higher or lower firing rate, nor were they associated with two discrete

499 sets of neurons, as in frontal eye fields (FEF) and superior colliculus, SC (Hanes and 
500 Carpenter, 1999; Pouget et al., 2017; Stuphorn et al., 2000). Instead, our results show

501 stopping correlates only when examining patterns found in ensembles of cells. This

502 finding suggests that encoding of stop signals may be more abstract than the coding in

503 more dorsal regions.

504 Overall, our results suggest one core function of OFC may be to generate an

505 abstract regulatory signal to feed into a cascade of downstream structures that ultimately

506 determine choice (Hunt and Hayden, 2017). In this way, it may be similar to other

507 regions, especially cingulate cortex (Blanchard et al., 2015b; Hillman and Bilkey, 2010;

508 Shenhav et al., 2013). In the context of economic choice, this signal will resemble a value

509 signal; in other cases it will correlate with other relevant task variables. This view is

510 consistent with the idea that choice and control processes both reflect a gradual

511 transformation occurring in a distributed manner across brain regions, rather than a

512 modular one (Balasubramani et al., 2018; Eisenreich et al., 2017; Hunt and Hayden,

513 2017) to study their neural codes. One benefit of view is that provides a basis for the

514 observed role of OFC and adjacent structures in self-control (Kable and Glimcher, 2007;

515 McClure et al., 2004).

516 The OFC is the major gateway by which sensory information enters into the

517 prefrontal cortex and is a major source of visceral information as well (Öngür and Price,

518 2000). It may therefore occupy an early position in PFC processing hierarchies

519 (Carmichael and Price, 1994; Fuster, 1988; Fuster, 2001; Rushworth et al., 2012;

520 Rushworth et al., 2011). These facts raise the possibility that OFC serves as a first (or at

521 least a relatively early) stage for computing preliminary executive signals that can affect 
bioRxiv preprint doi: https://doi.org/10.1101/304709; this version posted April 20, 2018. The copyright holder for this preprint (which was not certified by peer review) is the author/funder. All rights reserved. No reuse allowed without permission.

522 - but not determine behavior (Cavada et al., 2000; Ebitz and Hayden, 2016; Öngür and

523 Price, 2000; Wallis, 2007). 


\section{FIGURES}

525

526

527

528

529

530

531

532

533

534

535

536

537

538

539

540

541

542

543

544

545

546

547

548

549

550

551

552

553

554

555

556

557

558

559

560

561

562

563

564

565

566
Figure 1- subject behavior: task framework (A) stop signal task (B) economic choice task. Behavioral results for subject $\mathrm{J}$ are presented in panels $(\mathrm{C}, \mathrm{E}, \mathrm{G}, \mathrm{I}$, J) and for subject $T$ in (D, F, H, K, L) (C, D) reaction time distributions for various trial conditions of stop signal task (E, F) Inhibition function and accuracy of choices varied as a function of SSDs $(\mathbf{G}, \mathbf{H})$ reaction time distributions for various trial conditions of economic choice task. Previous trial had effects in reaction time behavior in $(\mathbf{I}, \mathbf{K})$ stop signal task and $(\mathbf{J}, \mathbf{L})$ economic choice task. Error bars represent SEM, and * denotes t-test significance with $p<0.05$. (M) recording site - Area 13 of OFC (scan from subject $J$ shown).

\section{Figure 2- overlapping sets of neurons across stopping and choice tasks:} Correlations between signed (A) task-related tuning coefficients, reward tuning coefficients in (B) post-go signal epoch (C) feedback epoch of stopping and economic choice tasks.

Figure 3- selectivity for stopping in sample neurons: Activity of example neurons during successful stopping, failed stopping and go trials are illustrated with respect to go signal in panels (A, B, E, F) and stop signal in (C, D) presentation time. Time from start of the go (stop) signal to SSRT is shaded all panels. Neuron in panel A shows significant difference in firing rates of successful and failed stopping trials before SSRT. Neuron in panel B shows difference even before the beginning of trial. Neuron in panel $\mathbf{C}$ is the same as panel $\mathbf{A}$, shows significant difference in firing rates of inhibition trials before stopping response time. Likewise, neuron in panel $\mathbf{D}$ shows difference around few msecs after SSRT. (E, F) Activity of example neurons in economic choice task: neuron in panel $\mathbf{E}$ is the same as panels $\mathbf{A}$ and $\mathbf{C}$. Its reward related activity after SSRT in the choice task parallels to that of stop signal task, and is positively correlated to the value. Neuron in panel F shows the opposite trend, and is negatively correlated to the reward value.

Figure 4- ensemble analysis inform about stopping: Performance of (A) prego signal (B) post-stop decoders to distinguish successful versus failed stopping pattern. Insets in both panels illustrate sample projections of decoder's output responses ( $\mathrm{Y}$ - and $\mathrm{Z}$-axes indicate the values of $\mathrm{O}_{1}$ and $\mathrm{O}_{2}$, for successful and failed stopping trials, respectively). Error bars indicate SEM, so non-overlap with the chance bar (horizontal dashed red line) is not sufficient to indicate statistical significance). Time points underneath through thick horizontal orange bars (and black tick marks) denote start time of $100 \mathrm{msec}$ boxcars having classification accuracy significantly above chance (i.e. $50 \%$ ) (chi-square test, $p<0.05$ ). 
567 Figure 5- unrelated reward and stopping codes: Correlations between

568 stopping and reward indices show no significant effect during $100 \mathrm{msec}$ in (A)

569 pre-go signal and (B) post-stop signal time period.

571 Figure 6- overlapping functional ensemble codes for stopping and choice:

572 Performance of decoders trained on (A) stopping task (B) choice task on post-

573 stop signal epoch (blue, magenta) and feedback (green, brown) epochs. Error

574 bars represent SEM. Time points underneath the thick horizontal highlighted bars

575 (blue, green, magenta, brown) of each result, as labelled in the legend, denote

576 the start time of $100 \mathrm{msec}$ boxcars having percent accuracies of classification

577 above chance of $50 \%$ (chi-square test, $p<0.05$ ). Red line indicates chance

578 (50\%) performance for both decoders.

579 


\section{METHODS}

\section{Subjects}

Two male rhesus macaques (Macaca mulatta, subject J, age 10, and subject T, age 5) served as subjects. All animal procedures were approved by the University Committee on Animal Resources at the University of Rochester and were designed and conducted in compliance with the Public Health Service's Guide for the Care and Use of Animals.

A Cilux recording chamber (Crist Instruments) was placed over the area 13 of 28.65 and $33.60 \mathrm{~mm}$ rostral to the interaural plane with varying depth. Position was verified by magnetic resonance imaging with the aid of a Brainsight system (Rogue Research Inc). Neuroimaging was performed at the Rochester Center for Brain Imaging, on a Siemens 3T MAGNETOM Trio Tim using $0.5 \mathrm{~mm}$ voxels. We confirmed recording locations by listening for characteristic sounds of white and grey matter during recording, which in all cases matched the loci indicated by the Brainsight system.

\section{Electrophysiological techniques}

Single electrodes (Frederick Haer \& Co., impedance range 0.8-4 MOhm) were lowered using a microdrive (NAN Instruments) until waveforms of between one and five neuron(s) were isolated. Individual action potentials were isolated on a Plexon system.

601 Neurons were selected for study solely based on the quality of isolation; we never

602 preselected based on task-related response properties.

\section{Eye tracking and reward delivery}

Eye position was sampled at $1,000 \mathrm{~Hz}$ by an infrared eye-monitoring camera

Task system (SR Research). Stimuli were controlled by a computer running MATLAB (Mathworks) with Psychtoolbox (Brainard and Vision, 1997) and Eyelink Toolbox (Cornelissen et al., 2002). A standard solenoid valve controlled the duration of water delivery. The relationship between solenoid open time and water volume was established and confirmed before, during, and after recording.

The stopping task is a measure of self-control that provides an alternative approach that avoids some of the limitations of intertemporal choice tasks (Hayden, 2016). The task followed standard stop signal paradigm (Hanes and Schall, 1995; Logan, 1994; Logan and Cowan, 1984). Subjects were placed in front of a computer monitor (1920x1080px) with black background. Following a brief (300 msec) central fixation on a white circle (radius 25px, Figure 1), the fixation spot disappeared on the appearance of eccentric saccade target (90px white square, 2.38 degrees, positioned at 288px in left or 
1632px in right of screen, 50\% chance). A go trial (67\% of trials, randomly selected) was indicated by a go signal which is the peripheral target, whereas a stop trial (33\% of trials, randomly selected) was indicated by an additional appearance of a stop signal - a central gray square (90px square, 2.38 degrees) delayed relative to the go signal presentation. Stop signal delays (SSD) in the task were set to stabilize at a delay causing approximately $50 \%$ successful stopping in average of all stop trials recorded till that moment of time in the day (SSD-50); SSDs were modulated through a staircase procedure with intervals of $16 \mathrm{msec}$. On go trials, subjects were rewarded for a saccade to the go signal and fixating on it for $200 \mathrm{msec}$; and on stop trials, subjects were rewarded for inhibiting their saccade to go signal and fixating at the stop signal for $400 \mathrm{msec}$. Water rewards were provided as feedback, and they were contingent on subject's performance. Rewards were always 125 $\mu \mathrm{l}$. The inter trial interval was $800 \mathrm{msec}$.

The economic choice task had a similar task framework to stop signal task, and they interleaved randomly in an interval of 1-3 trials. In go trials (random $67 \%$ of the total), a peripheral target called go offer (90px white square, 2.38 degrees, positioned at $288 \mathrm{px}$ in left or 1632px in right of the screen, 50\% chance) was presented, and it was randomly associated with low $(15 \mu \mathrm{l})$, medium $(125 \mu \mathrm{l})$, or high $(250 \mu \mathrm{l})$ reward offers, as indicated by yellow, blue and magenta colored squares, respectively. In this task, the go trials were named forced choice trials, and the go offer was called offer 1. In stop trials (random 33\% of the total)- called as choice trials, a center stop offer (offer 2, 90px square, 2.38 degrees) delayed with respect to the appearance of offer 1 was presented in addition. The offer 2 was also randomly associated with yellow, blue and magenta colors to indicate low, medium and high reward sizes. The offer 1 in stop trials was always in blue color to represent medium reward sized offer. This setup allowed the subject to make a choice through reward comparison in case of choice trials, and through a forced choice when only offer 1 was presented. All other parameters were the same as stop signal task.

\section{Behavioral analysis}

\section{Statistical methods}

Separate PSTH matrices were constructed by aligning spike rasters to the

660 calculated in $1 \mathrm{msec}$ bins but were generally analysed in longer epochs. Normalization 
661 procedure was carried out by subtracting the mean firing during inter-trial interval (ITI)

662 time period (baseline) and then by zscoring each neuron's data, and the normalized data

663 is used for decoder analysis presented in the manuscript. Alternatively, decoding was also

664 tested with just zscored data, and the results are presented in supplementary material. For

665 display, PSTHs were smoothened using $200 \mathrm{msec}$ running boxcars. Tests used in the

666 study include two sample t-test for parameteric analysis, Wilcoxon rank test for non-

667 parametric analysis, chi-square test for comparing decoder's classification accuracy

668 against baseline (50\% classification accuracy), Pearson correlation method for correlation

669 analysis. To compute population tuning, we picked neurons with significant $(\mathrm{p}<0.05)$

670 differences between successful and failed stopping trials using Wilcoxon rank test.

\section{Decoding analyses}

We chose a neural network based decoding technique because it could efficiently analyse population responses from frontal cortex that are highly multiplexed and nonlinear. To generate population activation states as input patterns for the decoding analysis, we first separated all trials of each neuron by trial conditions (successful and failed stopping trials). Then, we averaged the activity from randomly sampled 10 trials belonging to a condition, with replacement, to form activation state for a neuron in any particular time period. The averaged responses of all 96 neurons' were pooled to generate one population activation state for a particular trial condition and for a specific time period. 100 unique activation state patterns were used for the network training. $75 \%$ of the data was used for training and the rest was used for testing the network. The procedure is similar to that carried out by other studies (e.g., Mante et al., 2013; Pouget et al., 2000; Rigotti et al., 2013; Wang and Hayden, 2017).

The network used to study the stopping patterns had a single hidden layer with 100 hidden nodes, and 2 output nodes each representing one target condition for classification. The number of input nodes equal to the total number of neurons used for analysis $=96$ (from two subjects). The network weights were initialized to small random numbers between -0.01 and 0.01 .

The following back-propagation algorithm was used for training the decoders (Haykin and Network, 2004; Rumelhart et al., 1986; Rumelhart et al., 1988; Werbos, 1974). In the below, the input nodes are denoted by subscript, $k$, hidden nodes by subscript, $j$, and output nodes by subscript, $i$. Output error, $e$, associated with the network's response for the $p$ 'th input pattern was

$$
e_{\mathrm{i}}=\text { desired output }-\mathrm{y}_{\mathrm{i}}
$$

where $y_{\mathrm{i}}$ was the $i$ 'th output node response, and desired output was $1 / 0$ if the $i$ 'th output node was associated with target trial condition for the corresponding input pattern (e.g., successful stopping, failed stopping). Total output error over all input patterns was computed by,

$$
E=\sum_{p} E_{p}
$$

700 Network's objective was to minimize the squared output error (eqn. 1) for the $p$ 'th pattern 701 as denoted by eqn. (3).

$$
E_{p}=\frac{1}{2} \sum_{i} e_{i}^{2}
$$


and

Response of any node was a hyperbolic tangent function $(g)$ of slope $=5$ of the total input $\left(h_{\mathrm{i}}^{\mathrm{S}}\right)$ to it. The output node response, $y_{\mathrm{i}}$, as a function of its input was calculated as,

$$
y_{i}=g\left(h_{i}^{s}\right)
$$

where, net input $\left(h_{\mathrm{i}}^{\mathrm{S}}\right)$ to the output layer was,

$$
h_{i}^{s}=\sum_{j} w_{i j}^{s} V_{j}
$$

In the above, the weights, $w_{\mathrm{ij}}$, with superscript, $s$, indicate the second level of the network between hidden and output layer. $V_{\mathrm{j}}$ denoted the output of hidden layer, and it was represented as a function of net input to the hidden node $\left(h_{\mathrm{i}}^{\mathrm{f}}\right)$ as follows,

$$
V_{j}=g\left(h_{j}^{f}\right)
$$

$$
h_{j}^{f}=\sum_{k} w_{j k}^{f} x_{k}
$$

714

The superscript, $f$, in eqns. $(6,7)$ denote first level of the network between input and hidden layer, $w_{\mathrm{jk}}$ were their weights, and $x_{\mathrm{k}}$ was the input pattern to neural network.

where, Weight updates were proportional to the negative change in error for the $p$ 'th pattern, $E_{p}$, on change in weights. All updates happened trial by trial in the training phase. The update used at the second level was by eqn. (8), and that in the first level was by eqn. (10).

$$
\Delta w_{i j}^{s}=-\eta \frac{\partial E_{p}}{\partial w_{i j}^{s}}=\eta \delta_{i}^{s} V_{j}
$$

$$
\begin{aligned}
& \delta_{i}^{s}=e g^{\prime}\left(h_{i}^{s}\right) \\
& \Delta w_{j k}^{f}=\eta \delta_{j}^{f} x_{k}
\end{aligned}
$$

where,

$$
\delta_{j}^{f}=\sum_{i} \delta_{i}^{s} w_{i j}^{s} g^{\prime}\left(h_{j}^{f}\right)
$$

$\eta$ is the learning rate set to 0.001 for pre-go and post-stop signal decoder, and $g$ ' denotes first order derivative of hyperbolic tangent function.

We had two different decoders trained on data from 1) pre-go signal, 2) post-stop signal time periods to show OFC's active participation in stopping; the former worked on data aligned to presentation of go signal at time $=0$, and the latter worked on data aligned to stop signal. For pre-go decoder, the training data was population activation states generated on averaging the signal from the fixation epoch spanning $300 \mathrm{msec}$ before the presentation of go signal. For post-stop decoder, training data was generated on averaging the firing in the post-stop signal epoch. The entire network was run for $n=100$ instances with different random weight initializations to obtain average output performance. Training procedure in all instances converged to classification accuracy of above $80 \%$, and the converged weights at the end of training were used for testing of decoder. The testing data used were population activation states generated by averaging $100 \mathrm{msec}$ boxcars that slides with step size of $10 \mathrm{msec}$ (a total of 91 boxcars). 
Similarities in the functioning and generalization of pre-go and post-stop decoders

\section{$774 \quad$ Reward and stopping index}

775

776

777

778

were analysed by comparing their converged weights, as well as by comparing their classification accuracy. For cross validation, the similarity index (r-max) was computed by cross correlating converged hidden layer weight vectors (with zero lag) of two decoders of interest. The index was averaged across $n(=100)$ instances of networks with different weight initializations. The similarity index obtained from autocorrelating the weight vectors were used to statistically compare and cross-validate the results from cross correlation, and the results were significant using ttest (ttest, tstat $=210, \mathrm{p}<0.001$ ). Comparisons between classification accuracies of the two decoders, at pre-go signal time period or post-stop signal time period, were done by using t-test on average performances of the two decoders computed from $n$ instances (with different random weight initializations).

Cancellation time was defined by the size of test-boxcar window positioned at first instance of atleast four consecutive test-boxcars (100 msec window moving in intervals of $10 \mathrm{msec}$ ) in a row, whose performance was significantly higher than $50 \%$ using chi-square test $(\mathrm{p}<0.05)$. The method avoids false positives that otherwise appear by $99 \%$ chance when considering just any one single significant instance of 91 total boxcars. With simulations using markov chains, we found that at least 4 consecutive significant windows were needed in a row for the claim of significance with $p<0.001$; so the criteria to find at least 4 consecutive significant bins were used to find pre-go and post-stop decoder results as well as cancellation time. Average latency of cancellation signals to SSRT was found by subtracting SSRT of each subject from the mean cancellation time.

The decoders used for finding similarities between stopping and economic choices were similar to the above. The forced choice trials with offer 1 (accept) kind were chosen for analysis. The trials were divided into low and high value types, based on their reward magnitudes: the former type when the rewards were either low or medium, and the latter when the rewards were high, respectively. For this analysis, we also consider post-go signal decoder, similar to the post stop signal decoder except for its training on the post-go signal epoch from the presentation of go signal (in case of stop signal task) or offer 1 presentation (in case of economic choice task) till the reaction time. And, the feedback decoder was trained on the neural signals from the start to the end of feedback, for both the tasks. All decoders were tested using trials from economic choice task using boxcars of $100 \mathrm{msec}$ length moving in intervals of $10 \mathrm{msec}$.

Reward index for every neuron was measured by linearly regressing the firing at outcome epoch (between reaction time and feedback) to the received reward sizes in economic trials. The stopping index was measured as the difference in normalized firing rates (FR) of successful and failed stopping trials divided by their norm.

$$
\text { Stopping index }=\frac{F R_{\mathrm{sc}}-F R_{\mathrm{snc}}}{\sqrt{F R_{\mathrm{sc}}^{2}+F R_{\mathrm{snc}}^{2}}}
$$


Cross validation tests were performed to support the idea that we had sufficient data to detect an effect had it been there, and to suggest that our results of lack of a significant correlation between stopping and reward indices were statistically meaningful. For the cross validation analysis, all trials within a neuron were randomly separated to two groups, A and B. Stopping and reward index were computed for those two groups of each neuron. We performed correlations between stopping indices of groups A and B, and between reward indices of A and B. A total of $n(=100)$ random permutation instances were performed to generate different A and B sets. The test should ideally show high correlations between indices of $\mathrm{A}$ and $\mathrm{B}$ for any instance, and we indeed saw positive correlations between stopping-inde $x_{A}$ and stopping-index $x_{B}$, and similarly for reward-index $\mathrm{A}_{\mathrm{A}}$ and reward-index $\mathrm{x}_{\mathrm{B}}$. We confirmed that the actual correlation coefficient between stopping and reward indices in OFC fell within bottom $1 \%$ of the coefficients computed for $n$ instances of stopping-index $x_{A}$ and stopping-index $x_{\mathrm{B}}$. The above was also confirmed for $n$ coefficients for reward-index $\mathrm{A}_{\mathrm{A}}$ and reward-index $\mathrm{B}_{\mathrm{B}}$. The results showed 


\section{Results-A:}

\begin{tabular}{|c|c|c|c|c|}
\hline \multirow{2}{*}{\multicolumn{2}{|c|}{ Trial type }} & \multicolumn{2}{|c|}{$\begin{array}{l}\text { Reaction time distribution } \\
\text { medians } \\
(\mathrm{s})\end{array}$} & \multirow{2}{*}{$\begin{array}{c}\text { Encoding of value } \\
\text { In feedback epoch } \\
\text { Both subjects }\end{array}$} \\
\hline & & Subject $J$ & Subject $T$ & \\
\hline \multirow[t]{3}{*}{ Stopping task } & Go trials & $0.43 \pm 0.09$ & $0.27 \pm 0.12$ & \multirow{3}{*}{$\begin{array}{c}\quad r=0.27, \mathrm{p}<0.01 \\
\text { (unsigned: } \mathrm{r}=0.35, \mathrm{p}< \\
0.001 \text { ) }\end{array}$} \\
\hline & $\begin{array}{c}\text { Successful stopping } \\
\text { trials }\end{array}$ & $0.39 \pm 0.09$ & $0.25 \pm 0.08$ & \\
\hline & Failed stopping trials & $0.25 \pm 0.20$ & $0.15 \pm 0.22$ & \\
\hline \multirow{3}{*}{$\begin{array}{c}\text { Economic choice } \\
\text { task }\end{array}$} & Forced choice trials & $0.41 \pm 0.12$ & $0.27 \pm 0.13$ & \multirow[t]{3}{*}{$\mathrm{r}=-1.5342, \mathrm{p}=0.0025$} \\
\hline & Choose offer 2 trials & $0.46 \pm 0.12$ & $0.25 \pm 0.10$ & \\
\hline & Choose offer 1 trials & $0.28 \pm 0.19$ & $0.20 \pm 0.23$ & \\
\hline
\end{tabular}

\begin{tabular}{|c|c|c|c|}
\hline \multicolumn{2}{|c|}{$\begin{array}{c}\text { Economic task } \\
\text { Variables }\end{array}$} & Subject $~$ & Subject T \\
\hline $\begin{array}{c}\text { Reaction times versus value } \\
\text { of offers }\end{array}$ & $\begin{array}{c}\text { Forced choice } \\
\text { trials- offer 1 }\end{array}$ & $\begin{array}{c}\text { Pearson correlation, } \mathrm{r}=0.52, \\
\mathrm{p}<0.001\end{array}$ & $\mathrm{r}=0.43, \mathrm{p}<0.001$ \\
\cline { 2 - 4 } & $\begin{array}{c}\text { Choice trials } \\
\text { offer 2 }\end{array}$ & $\mathrm{r}=0.29, \mathrm{p}=0.003$ & $\mathrm{r}=0.27, \mathrm{p}<0.001$ \\
\hline
\end{tabular}




\begin{tabular}{|c|c|c|c|}
\hline $\begin{array}{l}\text { Preference for offer } 1 \text { than } \\
\text { offer } 2\end{array}$ & Choice trials & $\begin{array}{c}57.6 \% \\
\text { (chi-square test, chi-square }= \\
5.78, \mathrm{p}=0.016)\end{array}$ & $\begin{array}{c}56.25 \% \\
(\text { chi-square stat }= \\
3.38, \mathrm{p}=0.066)\end{array}$ \\
\hline $\begin{array}{l}\text { Preference for offer } 1 \text { versus } \\
\text { stop signal delay }\end{array}$ & Choice trials & $\begin{array}{l}\text { slope of psychometric } \\
\text { sigmoidal fit }=5.53\end{array}$ & Slope $=6.93$ \\
\hline $\begin{array}{c}\text { Accuracy (choosing best } \\
\text { offer) }\end{array}$ & Choice trials & $89.25 \%$ & $89.8 \%$ \\
\hline $\begin{array}{c}\text { Accuracy versus stop signal } \\
\text { delay }\end{array}$ & Choice trials & $\begin{array}{l}\text { slope of psychometric } \\
\text { sigmoidal fit }=-4.93\end{array}$ & Slope $=-5.3$ \\
\hline \multirow[t]{2}{*}{ Neuronal tuning for rewards } & Offer 1 & $21.15 \%$ & $9.09 \%$ \\
\hline & Offer 2 & $11.5 \%$ & $9.09 \%$ \\
\hline
\end{tabular}

800
Both subjects showed behavioral effects in the reaction times of successful stoppings as a function of previous trial conditions (Figure 1). Successful stopping trials were shorter when following a successful stopping trial (subject $\mathrm{J}: \mathrm{N}=328$, subject $\mathrm{T}: \mathrm{N}=357$ ) as opposed to following a failed stopping (subject $\mathrm{J}: \mathrm{N}=111$, subject $\mathrm{T}: \mathrm{N}=60$ ). The statistical t-test on data for subject $\mathrm{J}$ was $360 \mathrm{msec}$ shorter, with $\mathrm{t}$-stat $=11.33, \mathrm{p}<0.0001$ and for subject T was: $290 \mathrm{msec}$ shorter, $\mathrm{t}-\mathrm{stat}=11.88, \mathrm{p}<0.0001)$. Similarly, successful stopping trials were shorter when following a go trial (subject $\mathrm{J}: \mathrm{N}=833$, subject $\mathrm{T}: \mathrm{N}=$ 862 ) as opposed to following a failed stopping trial (subject J: $310 \mathrm{msec}$ shorter, $\mathrm{t}$-stat $=$ 9.608, $\mathrm{p}<0.0001$ and subject T: 210 msec shorter, t-stat $=7.72, \mathrm{p}<0.0001$ ). We found a choice of reject trials (choice of offer 2) were shorter when following another reject trial compared to an accept trial (subject J: $0.28 \mathrm{~s}$, tstat $=8.47, \mathrm{p}<0.001$, Figure K; subject T: $0.31 \mathrm{~s}$, tstat $=9.49, \mathrm{p}<0.001$, Figure 1). Consequent reject trials were also shorter than following an accept trial (subject J: $0.1 \mathrm{~s}$, tstat $=5.96, \mathrm{p}<0.001$, Figure K; subject T: 0.18 , tstat $=4.97, \mathrm{p}<0.001)$. Accept trials were generally longer after following reject trials than following another accept trial (subject J: $0.05 \mathrm{~s}$, tstat $=3.41, \mathrm{p}<0.001$, Figure $\mathrm{K}$; subject $\mathrm{T}$ : tstat $=2.06, \mathrm{p}=0.039$ ).

The proportion of neurons that showed positive task-related tuning during successful stopping when regressed against baseline were $31.25 \%$, while that showed negative tuning were $39.58 \%$. Similarly, the proportion that showed positive task-related tuning during failed stopping activity when regressed against baseline were $34.38 \%$, while that showed negative tuning were $34.38 \%$. In economic choice task, the percent of neurons that showed positive feedback tuning when regressed against baseline were $45.83 \%$, while that showed negative tuning were $51.04 \%$. Overall, the tuning for success and failure of stopping significantly correlated with the feedback tuning of economic choice task (Pearson correlation, $\mathrm{r}=0.62, \mathrm{p}<0.001$ ). 


\section{Results-B:}

\section{Population averages provide weak information about stopping}

Analysis of single neurons did not provide strong evidence for a role for OFC in stopping. The percent of neurons that individually distinguish successful and failed stopping trials (regardless of sign) was $8.43 \%$ during the $100 \mathrm{msec}$ post-stop signal time period, and was $10.50 \%$ during the $100 \mathrm{msec}$ pre-go signal time period. (These epochs were selected before analysis in order to reduce the likelihood of p-hacking). These proportions were not significantly greater than chance in either of the two key epochs (chi-square stat $=1.22, \mathrm{p}=0.26$ in the post-stop signal time period; chi-square stat $=1.8$, $\mathrm{p}=0.17$ in the pre-go signal time period). This lack of a detectable effect does not imply that a correlation between stopping and unit activity in OFC does not exist; rather it suggests that if it does exist it is too weak to detect using conventional methods that focus on single neurons in a sample of the size we collected.

We next tested whether successful and failed stopping trials have a consistent sign of effect on firing rates. The percent of significantly positive cells (successful $>$ failed) was $5.40 \%$, and wasn't significantly different from the percent of significantly negative ( successful $<$ failed) cells $(3.03 \%$ chi-square test, chi-square stat $=0.52, \mathrm{p}=0.47)$ in the post-stop signal period. The difference in the sizes of the two cell classes was also not significant before the start of trial at the pre-go signal time period (significantly positive cells $7.55 \%$, significantly negative cells $2.95 \%$, chi square $=2.40, \mathrm{p}=0.12$ ).

Next we looked at grand averages of populations of neurons. We observed no difference between successful and failed stopping trials either after the stop signal or before the beginning of trial. Specifically, during the post-stop signal time period, responses were slightly less for successful than failed stopping in subject $\mathbf{J}$ (average of 0.3 spikes/sec, $\mathrm{p}=0.6$ ); the opposite pattern was observed in subject $\mathrm{T}$ (average of 0.52 spikes/sec, $\mathrm{p}=0.53$ ). Neither effect was statistically significant. Thus, these results suggest that conventional population averages don't reveal information about the pattern of stopping. Together these analyses indicate that, if stopping correlates exist in OFC, they are of a different form than they take in regions like FEF and SC. 

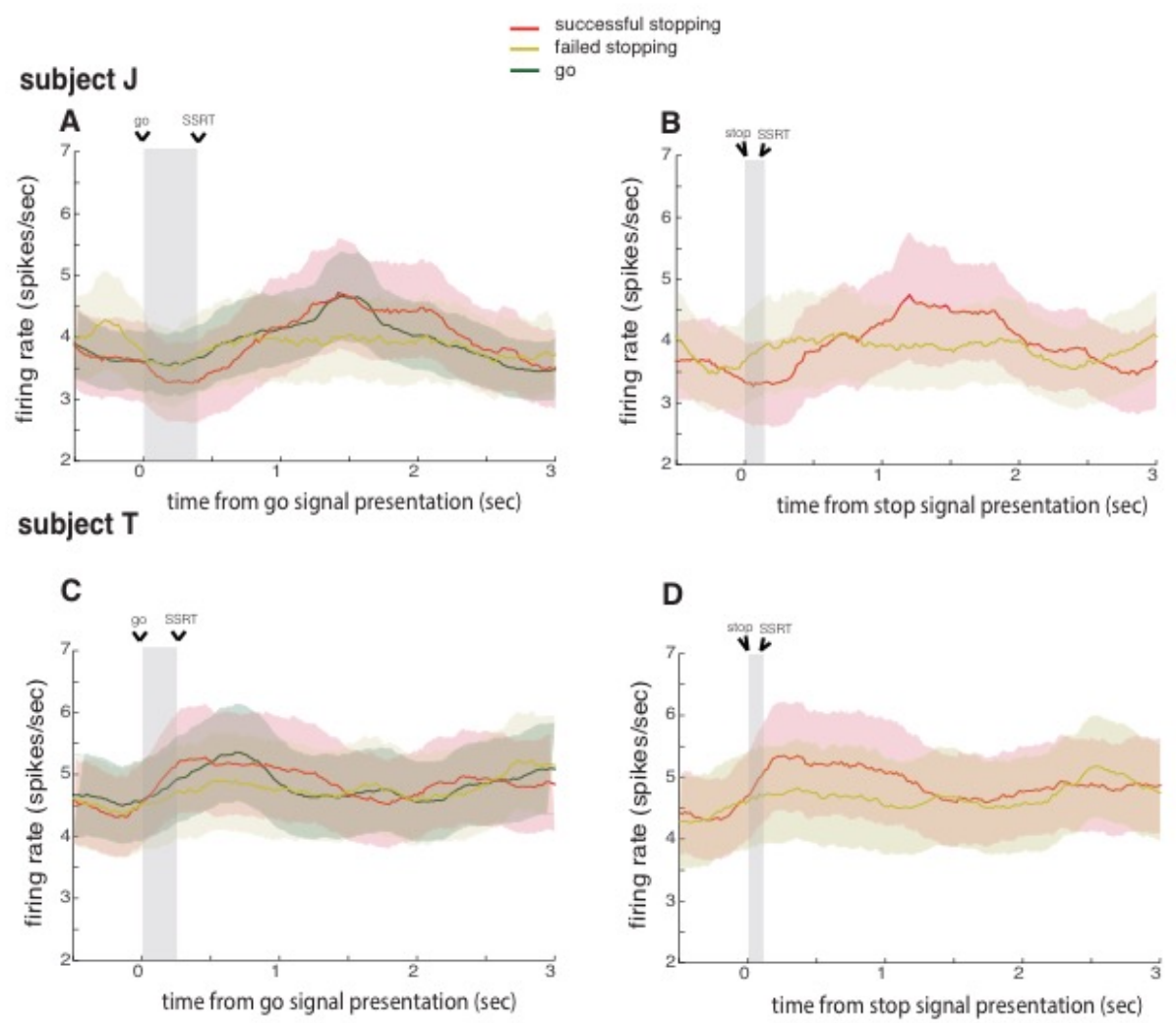

Figure- population averages provide weak information about stopping:

861 C) go signal presentation and (B, D) stop signal presentation, for subjects $\mathrm{J}$ and

862 T. Time from start of the go (stop) signal to SSRT is shaded in panels $A$ and $C$ ( $B$ and D). Data for all SSDs are averaged to present successful and failed stopping trials. Error bars denote SEM. They don't reveal significant information about the

865 pattern of stopping. 
878
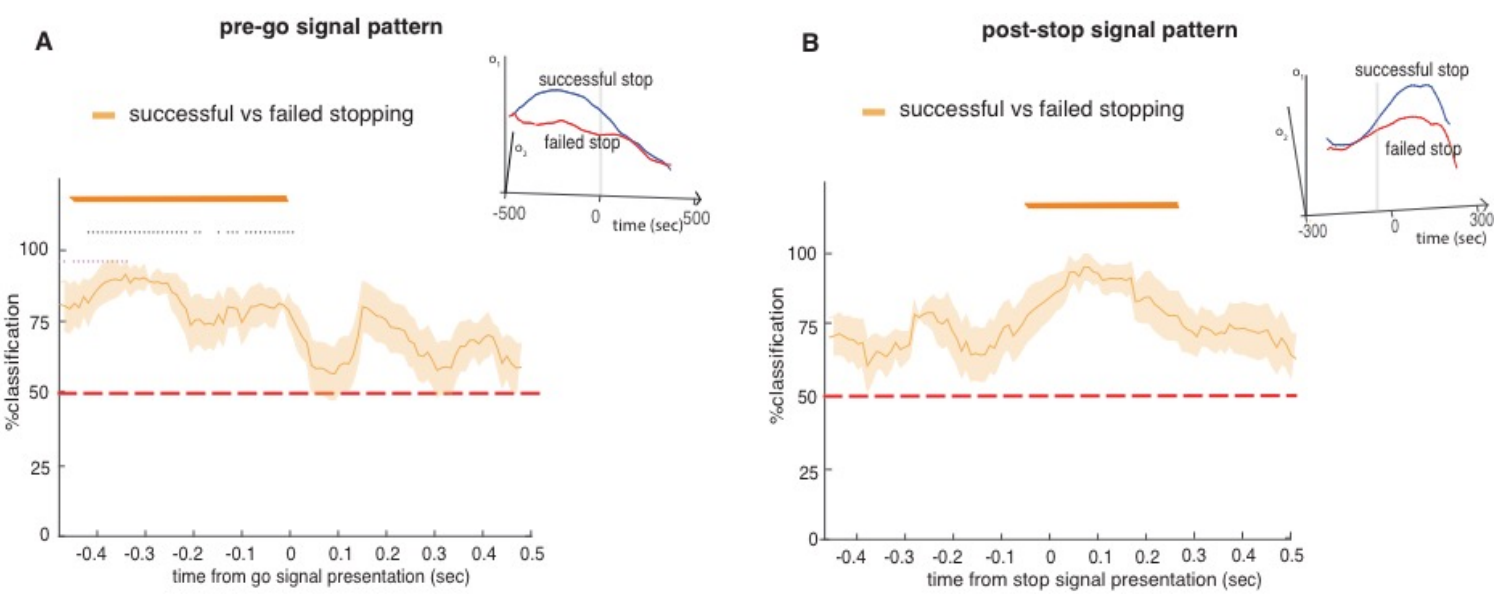

880 Figure- Post-stop and pre-go decoding for subjects $\mathbf{J}$ and $\mathbf{T}$ with zscored

881 data (normalization): Both post stop signal and pre- go signal decoder was able

882 to classify success of stopping significantly above chance (see Methods for

883 specific use of chi-square tests to quantify significance) before SSRT and go

884 signal presentation, respectively, and chi-square tests were used for finding their

885 significance with $p<0.05$, see Methods). Results suggest that successful

886 differentiation of stopping codes can be obtained irrespective of the normalization

887 methods used in the study (In the manuscript, Normalization procedure was

888 carried out by subtracting the mean firing during inter-trial interval (ITI) time

889 period (baseline) and then by zscoring each neuron's data, and the normalized

890 data is used for decoder analysis).

891 
subject J

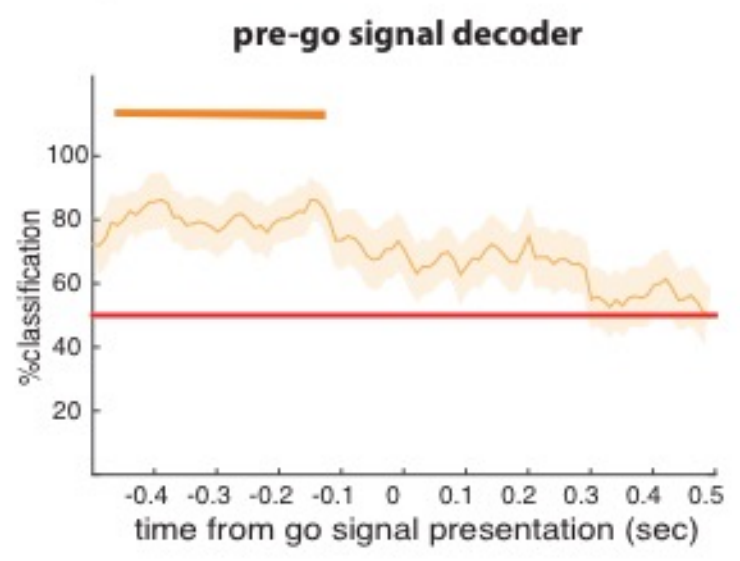

- successful vs failed stopping

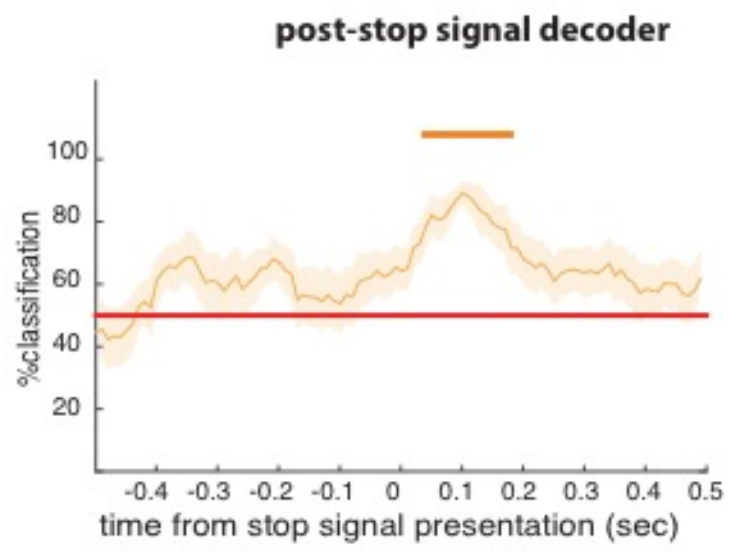

subject T

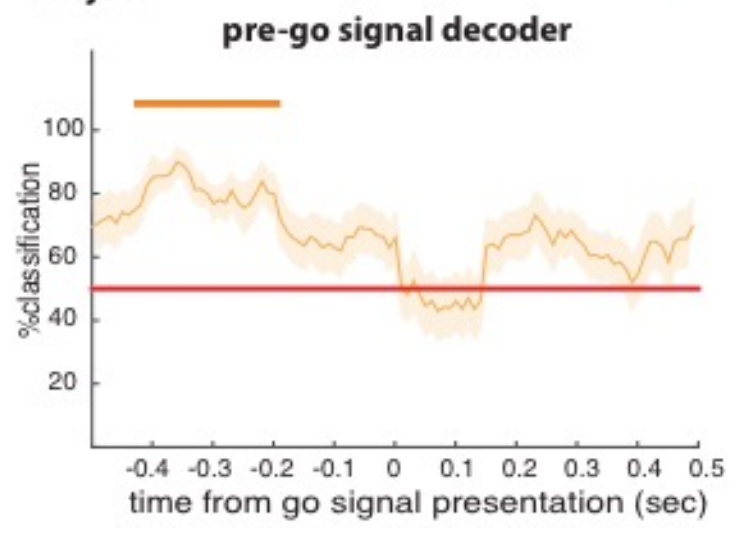

successful vs failed stopping

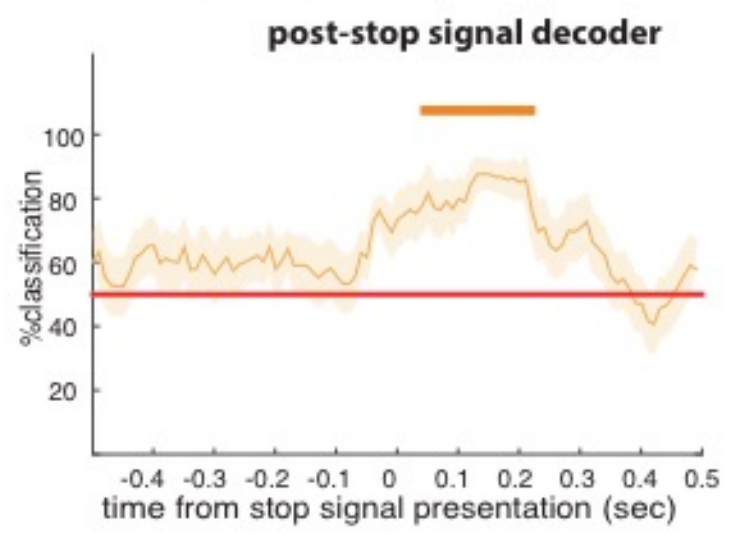

894

895

896

897

898

899

900

901

902

903

904

905

906

907

908

909
Figure- Post-stop and pre-go decoding for subjects $\mathrm{J}$ and T: The post stop signal decoder was able to classify success of stopping significantly above chance (see Methods for specific use of chi-square tests to quantify significance) in a time period ranging from $40 \mathrm{msec}$ to $170 \mathrm{msec}$ for subject $\mathrm{J}$, and 40 to 220 msec for subject $T$, respectively after the stop signal (these times indicate the beginning of $100 \mathrm{msec}$ boxcars, and chi-square tests were used for finding their significance with $p<0.05$, see Methods). The first significant bin was therefore of window size $40-140 \mathrm{msec}$, that led to average cancellation time as $90 \mathrm{msec}$. It preceded the average stopping response by $50 \mathrm{msec}$ in subject $\mathrm{J}$, and by 30 msec in subject T, suggesting OFC's responses may precede the stopping response. In Pre-go signal decoder, for subject $J$, high accuracy of decoding was found during the time periods $460 \mathrm{msec}$ to $120 \mathrm{msec}$ before the appearance of go signal. Likewise, it was $420 \mathrm{msec}$ to $200 \mathrm{msec}$ in subject T. 


\section{REFERENCES}

911 Aron, A.R. (2007). The neural basis of inhibition in cognitive control. The neuroscientist 912 13, 214-228.

913 Aron, A.R., and Poldrack, R.A. (2006). Cortical and subcortical contributions to stop

914

915

916

917

918

919

920 signal response inhibition: role of the subthalamic nucleus. Journal of Neuroscience 26, 2424-2433.

Balasubramani, P.P., Moreno-Bote, R., and Hayden, B. (2018). Using a simple neural network to delineate some principles of distributed economic choice. Frontiers in Computational Neuroscience 12, 22.

Berkman, E., Hutcherson, C., Livingston, J.L., Kahn, L.E., and Inzlicht, M. (2016). Selfcontrol as value-based choice.

Blanchard, T.C., and Hayden, B.Y. (2015). Monkeys are more patient in a foraging task than in a standard intertemporal choice task. PloS one 10, e0117057.

Blanchard, T.C., Hayden, B.Y., and Bromberg-Martin, E.S. (2015a). Orbitofrontal cortex uses distinct codes for different choice attributes in decisions motivated by curiosity. Neuron 85, 602-614.

Blanchard, T.C., Strait, C.E., and Hayden, B.Y. (2015b). Ramping ensemble activity in dorsal anterior cingulate neurons during persistent commitment to a decision. Journal of neurophysiology 114, 2439-2449.

Brainard, D.H., and Vision, S. (1997). The psychophysics toolbox. Spatial vision 10, 433-436.

Braver, T.S. (2012). The variable nature of cognitive control: a dual mechanisms framework. Trends in cognitive sciences 16, 106-113.

Braver, T.S., Gray, J.R., and Burgess, G.C. (2007). Explaining the many varieties of working memory variation: Dual mechanisms of cognitive control. Variation in working memory, 76-106.

Bryden, D.W., and Roesch, M.R. (2015). Executive control signals in orbitofrontal cortex during response inhibition. The Journal of Neuroscience 35, 3903-3914.

Carmichael, S., and Price, J. (1994). Architectonic subdivision of the orbital and medial prefrontal cortex in the macaque monkey. Journal of Comparative Neurology 346, 366-402.

Cavada, C., Compañy, T., Tejedor, J., Cruz-Rizzolo, R.J., and Reinoso-Suárez, F. (2000). The anatomical connections of the macaque monkey orbitofrontal cortex. A review. Cerebral Cortex 10, 220-242.

Chen, X., Scangos, K.W., and Stuphorn, V. (2010). Supplementary motor area exerts proactive and reactive control of arm movements. Journal of Neuroscience 30 , 14657-14675.

Chikazoe, J., Jimura, K., Hirose, S., Yamashita, K.-i., Miyashita, Y., and Konishi, S. (2009). Preparation to inhibit a response complements response inhibition during performance of a stop-signal task. Journal of Neuroscience 29, 1587015877.

Chudasama, Y., Kralik, J., and Murray, E. (2006). Rhesus monkeys with orbital prefrontal cortex lesions can learn to inhibit prepotent responses in the reversed reward contingency task. Cerebral Cortex 17, 1154-1159.

Cisek, P. (2012). Making decisions through a distributed consensus. Current opinion in neurobiology 22, 927-936.

Cisek, P., and Kalaska, J.F. (2010). Neural mechanisms for interacting with a world full of action choices. Annual review of neuroscience 33, 269-298. 
958

959

960

961

962

963

964

965

966

967

968

969

970

971

972

973

974

975

976

977

978

979

980

981

982

983

984

985

986

987

988

989

990

991

992

993

994

995

996

997

998

999

1000

1001

1002

1003

1004

1005

Cisek, P., and Pastor-Bernier, A. (2014). On the challenges and mechanisms of embodied decisions. Phil Trans R Soc B 369, 20130479.

Dias, R., Robbins, T., and Roberts, A. (1996). Dissociation in prefrontal cortex of affective and attentional shifts. Nature 380, 69-72.

Eagle, D.M., Baunez, C., Hutcheson, D.M., Lehmann, O., Shah, A.P., and Robbins, T.W. (2007). Stop-signal reaction-time task performance: role of prefrontal cortex and subthalamic nucleus. Cerebral cortex 18, 178-188.

Ebitz, R.B., and Hayden, B.Y. (2016). Dorsal anterior cingulate: a Rorschach test for cognitive neuroscience. Nature neuroscience 19, 1278.

Eisenreich, B.R., Akaishi, R., and Hayden, B.Y. (2017). Control without Controllers: Toward a Distributed Neuroscience of Executive Control. Journal of Cognitive Neuroscience.

Feierstein, C.E., Quirk, M.C., Uchida, N., Sosulski, D.L., and Mainen, Z.F. (2006). Representation of spatial goals in rat orbitofrontal cortex. Neuron 51, 495-507.

Freidin, E., Aw, J., and Kacelnik, A. (2009). Sequential and simultaneous choices: Testing the diet selection and sequential choice models. Behavioural processes $80,218-223$.

Fuster, J.M. (1988). Prefrontal cortex. In Comparative neuroscience and neurobiology (Springer), pp. 107-109.

Fuster, J.n.M. (2001). The prefrontal cortex-an update: time is of the essence. Neuron 30, 319-333.

Ghods-Sharifi, S., Haluk, D.M., and Floresco, S.B. (2008). Differential effects of inactivation of the orbitofrontal cortex on strategy set-shifting and reversal learning. Neurobiology of learning and memory 89, 567-573.

Grattan, L.E., and Glimcher, P.W. (2014). Absence of spatial tuning in the orbitofrontal cortex. PloS one 9, e112750.

Hampshire, A., and Sharp, D.J. (2015). Contrasting network and modular perspectives on inhibitory control. Trends in cognitive sciences 19, 445-452.

Hanes, D.P., and Carpenter, R. (1999). Countermanding saccades in humans. Vision research 39, 2777-2791.

Hanes, D.P., Patterson, W.F., and Schall, J.D. (1998). Role of frontal eye fields in countermanding saccades: visual, movement, and fixation activity. Journal of Neurophysiology 79, 817-834.

Hanes, D.P., and Schall, J.D. (1995). Countermanding saccades in macaque. Visual neuroscience 12, 929-937.

Hayden, B.Y. (2018). Economic choice: the foraging perspective. Current Opinion in Behavioral Sciences 24, 1-6.

Hayden, B.Y., (2016). Time discounting and time preference in animals: A critical review. Psychon. Bull. Rev. 23, 39-53. https://doi.org/10.3758/s13423-015-0879-3

Hayden, B.Y., and Moreno-Bote, R. (2018). A neuronal theory of sequential economic choice. Brain and Neuroscience Advances 2, 2398212818766675.

Haykin, S., and Network, N. (2004). A comprehensive foundation. Neural Networks 2, 41.

Hillman, K.L., and Bilkey, D.K. (2010). Neurons in the rat anterior cingulate cortex dynamically encode cost-benefit in a spatial decision-making task. Journal of Neuroscience 30, 7705-7713.

Horn, N., Dolan, M., Elliott, R., Deakin, J., and Woodruff, P. (2003). Response inhibition and impulsivity: an fMRI study. Neuropsychologia 41, 1959-1966. 
1006

1007

1008

1009

1010

1011

1012

1013

1014

1015

1016

1017

1018

1019

1020

1021

1022

1023

1024

1025

1026

1027

1028

1029

1030

1031

1032

1033

1034

1035

1036

1037

1038

1039

1040

1041

1042

1043

1044

1045

1046

1047

1048

1049

1050

1051

1052

1053

1054
Hunt, L.T., and Hayden, B.Y. (2017). A distributed, hierarchical and recurrent framework for reward-based choice. Nat Rev Neurosci 18, 172-182.

lacono, W.G., Malone, S.M., and McGue, M. (2008). Behavioral disinhibition and the development of early-onset addiction: common and specific influences. Annu Rev Clin Psychol 4, 325-348.

Inzlicht, M., Schmeichel, B.J., and Macrae, C.N. (2014). Why self-control seems (but may not be) limited. Trends in cognitive sciences 18, 127-133.

Ito, S., Stuphorn, V., Brown, J.W., and Schall, J.D. (2003). Performance monitoring by the anterior cingulate cortex during saccade countermanding. Science 302, $120-$ 122.

Kable, J.W., and Glimcher, P.W. (2007). The neural correlates of subjective value during intertemporal choice. Nature neuroscience 10, 1625.

Kacelnik, A., Vasconcelos, M., Monteiro, T., and Aw, J. (2011). Darwin's "tug-of-war" vs. starlings' "horse-racing": how adaptations for sequential encounters drive simultaneous choice. Behavioral Ecology and Sociobiology 65, 547-558.

Krajbich, I., Armel, C., and Rangel, A. (2010). Visual fixations and the computation and comparison of value in simple choice. Nature neuroscience 13, 1292.

Lara, A.H., Kennerley, S.W., and Wallis, J.D. (2009). Encoding of gustatory working memory by orbitofrontal neurons. Journal of Neuroscience 29, 765-774.

Lim, S.-L., O'Doherty, J.P., and Rangel, A. (2011). The decision value computations in the vmPFC and striatum use a relative value code that is guided by visual attention. Journal of Neuroscience 31, 13214-13223.

Logan, G.D. (1994). On the ability to inhibit thought and action: A users' guide to the stop signal paradigm.

Logan, G.D., and Cowan, W.B. (1984). On the ability to inhibit thought and action: A theory of an act of control. Psychological review 91, 295.

Logan, G.D., Yamaguchi, M., Schall, J.D., and Palmeri, T.J. (2015). Inhibitory control in mind and brain 2.0: blocked-input models of saccadic countermanding. Psychological review 122, 115.

Majid, D.A., Cai, W., Corey-Bloom, J., and Aron, A.R. (2013). Proactive selective response suppression is implemented via the basal ganglia. Journal of Neuroscience 33, 13259-13269.

Mansouri, F.A., Buckley, M.J., and Tanaka, K. (2014). The essential role of primate orbitofrontal cortex in conflict-induced executive control adjustment. Journal of Neuroscience 34, 11016-11031.

Mante, V., Sussillo, D., Shenoy, K.V., and Newsome, W.T. (2013). Context-dependent computation by recurrent dynamics in prefrontal cortex. nature 503, 78.

McClure, S.M., Laibson, D.I., Loewenstein, G., and Cohen, J.D. (2004). Separate neural systems value immediate and delayed monetary rewards. Science 306, 503507.

Meyer, H.C., and Bucci, D.J. (2016). Imbalanced activity in the orbitofrontal cortex and nucleus accumbens impairs behavioral inhibition. Current biology 26, 28342839.

Miller, E.K. (2000). The prefontral cortex and cognitive control. Nature reviews neuroscience 1, 59.

Miller, E.K., and Cohen, J.D. (2001). An integrative theory of prefrontal cortex function. Annual review of neuroscience 24, 167-202.

Mishkin, M. (1964). Perseveration of central sets after frontal lesions in monkeys. The frontal granular cortex and behavior, 219-241. 
1055

1056

1057

1058

1059

1060

1061

1062

1063

1064

1065

1066

1067

1068

1069

1070

1071

1072

1073

1074

1075

1076

1077

1078

1079

1080

1081

1082

1083

1084

1085

1086

1087

1088

1089

1090

1091

1092

1093

1094

1095

1096

1097

1098

1099

1100

1101

1102

Nestler, E.J., Barrot, M., DiLeone, R.J., Eisch, A.J., Gold, S.J., and Monteggia, L.M. (2002). Neurobiology of depression. Neuron 34, 13-25.

Ojeda, A., Murphy, R.A., and Kacelnik, A. (2018). Paradoxical choice in rats: subjective valuation and mechanism of choice. Behavioural processes.

Öngür, D., and Price, J. (2000). The organization of networks within the orbital and medial prefrontal cortex of rats, monkeys and humans. Cerebral cortex 10, 206219.

Padoa-Schioppa, C. (2011). Neurobiology of economic choice: a good-based model. Annual review of neuroscience 34, 333.

Padoa-Schioppa, C. (2013). Neuronal origins of choice variability in economic decisions. Neuron 80, 1322-1336.

Padoa-Schioppa, C., and Assad, J.A. (2006). Neurons in the orbitofrontal cortex encode economic value. Nature 441, 223-226.

Pirrone, A., Azab, H., Hayden, B., Stafford, T., and Marshall, J.A. (2017). Evidence for the speed-value trade-off: human and monkey decision making is magnitude sensitive. Decision (in press).

Pouget, A., Dayan, P., and Zemel, R. (2000). Information processing with population codes. Nature Reviews Neuroscience 1, 125.

Pouget, P., Murthy, A., and Stuphorn, V. (2017). Cortical control and performance monitoring of interrupting and redirecting movements. Phil Trans R Soc B 372, 20160201.

Raghuraman, A.P., and Padoa-Schioppa, C. (2014). Integration of multiple determinants in the neuronal computation of economic values. Journal of Neuroscience 34, 11583-11603.

Rich, E., Stoll, F., and Rudebeck, P. (2017). Linking dynamic patterns of neural activity in orbitofrontal cortex with decision making. Current opinion in neurobiology 49 , 24-32.

Rich, E.L., and Wallis, J.D. (2016). Decoding subjective decisions from orbitofrontal cortex. Nature neuroscience 19, 973.

Rigotti, M., Barak, O., Warden, M.R., Wang, X.-J., Daw, N.D., Miller, E.K., and Fusi, S. (2013). The importance of mixed selectivity in complex cognitive tasks. Nature 497, 585-590.

Roberts, A., and Wallis, J. (2000). Inhibitory control and affective processing in the prefrontal cortex: neuropsychological studies in the common marmoset. Cerebral Cortex 10, 252-262.

Roesch, M.R., Taylor, A.R., and Schoenbaum, G. (2006). Encoding of time-discounted rewards in orbitofrontal cortex is independent of value representation. Neuron 51, 509-520.

Rudebeck, P.H., and Murray, E.A. (2014). The orbitofrontal oracle: cortical mechanisms for the prediction and evaluation of specific behavioral outcomes. Neuron 84 , 1143-1156.

Rumelhart, D., McClelland, J., and Williams, R. (1986). Parallel recognition in modern computers. processing: Explorations in the microstructure of cognition 1.

Rumelhart, D.E., McClelland, J.L., and Group, P.R. (1988). Parallel distributed processing, Vol 1 (IEEE).

Rushworth, M.F., Kolling, N., Sallet, J., and Mars, R.B. (2012). Valuation and decisionmaking in frontal cortex: one or many serial or parallel systems? Current opinion in neurobiology $22,946-955$. 
1103 Rushworth, M.F., Noonan, M.P., Boorman, E.D., Walton, M.E., and Behrens, T.E.

1104

1105

1106

1107

1108

1109

1110

1111

1112

1113

1114

1115

1116

1117

1118

1119

1120

1121

1122

1123

1124

1125

1126

1127

1128

1129

1130

1131

1132

1133

1134

1135

1136

1137

1138

1139

1140

1141

1142

1143

1144

1145

1146

1147

1148

1149
(2011). Frontal cortex and reward-guided learning and decision-making. Neuron 70, 1054-1069.

Sakagami, M., and Pan, X. (2007). Functional role of the ventrolateral prefrontal cortex in decision making. Current opinion in neurobiology 17, 228-233.

Schall, J.D. (1991). Neuronal activity related to visually guided saccades in the frontal eye fields of rhesus monkeys: comparison with supplementary eye fields. Journal of neurophysiology 66, 559-579.

Schall, J.D. (2001). Neural basis of deciding, choosing and acting. Nature Reviews Neuroscience 2, 33-42.

Schall, J.D., Stuphorn, V., and Brown, J.W. (2002). Monitoring and control of action by the frontal lobes. Neuron 36, 309-322.

Schoenbaum, G., Roesch, M.R., Stalnaker, T.A., and Takahashi, Y.K. (2009). A new perspective on the role of the orbitofrontal cortex in adaptive behaviour. Nature Reviews Neuroscience 10, 885-892.

Schoenbaum, G., Setlow, B., Nugent, S.L., Saddoris, M.P., and Gallagher, M. (2003). Lesions of orbitofrontal cortex and basolateral amygdala complex disrupt acquisition of odor-guided discriminations and reversals. Learning \& Memory 10, 129-140.

Schoenbaum, G., Takahashi, Y., Liu, T.L., and McDannald, M.A. (2011). Does the orbitofrontal cortex signal value? Annals of the New York Academy of Sciences 1239, 87-99.

Schultz, W. (2000). Multiple reward signals in the brain. Nature reviews Neuroscience 1, 199.

Shapiro, M.S., Siller, S., and Kacelnik, A. (2008). Simultaneous and sequential choice as a function of reward delay and magnitude: normative, descriptive and processbased models tested in the European starling (Sturnus vulgaris). Journal of Experimental Psychology: Animal Behavior Processes 34, 75.

Shenhav, A. (2017). The perils of losing control: Why self-control is not just another value-based decision. Psychological Inquiry

Shenhav, A., Botvinick, M.M., and Cohen, J.D. (2013). The expected value of control: an integrative theory of anterior cingulate cortex function. Neuron 79, 217-240.

Sleezer, B.J., Castagno, M.D., and Hayden, B.Y. (2016). Rule encoding in orbitofrontal cortex and striatum guides selection. Journal of Neuroscience 36, 11223-11237.

Sleezer, B.J., LoConte, G.A., Castagno, M.D., and Hayden, B.Y. (2017). Neuronal responses support a role for orbitofrontal cortex in cognitive set reconfiguration. European Journal of Neuroscience 45, 940-951.

Stalnaker, T.A., Cooch, N.K., and Schoenbaum, G. (2015). What the orbitofrontal cortex does not do. Nature Neuroscience 18, 620-627.

Stephens, D.W., and Anderson, D. (2001). The adaptive value of preference for immediacy: when shortsighted rules have farsighted consequences. Behavioral Ecology 12, 330-339.

Stephens, D.W., and Krebs, J.R. (1986). Foraging theory (Princeton University Press).

Strait, C.E., Blanchard, T.C., and Hayden, B.Y. (2014). Reward value comparison via mutual inhibition in ventromedial prefrontal cortex. Neuron 82, 1357-1366.

Strait, C.E., Sleezer, B.J., and Hayden, B.Y. (2015). Signatures of value comparison in ventral striatum neurons. PLoS Biol 13, e1002173. 

Neuronal selectivity for spatial positions of offers and choices in five reward regions. Journal of neurophysiology 115(3):1098-111.

Stuphorn, V., Bauswein, E., and Hoffmann, K.-P. (2000). Neurons in the primate superior colliculus coding for arm movements in gaze-related coordinates. Journal of Neurophysiology 83, 1283-1299.

Stuphorn, V., Brown, J.W., and Schall, J.D. (2010). Role of supplementary eye field in saccade initiation: executive, not direct, control. Journal of neurophysiology 103 , 801-816.

Stuphorn, V., and Emeric, E.E. (2012). Proactive and reactive control by the medial frontal cortex. Frontiers in Neuroengineering 5.

Sugrue, L.P., Corrado, G.S., and Newsome, W.T. (2005). Choosing the greater of two goods: neural currencies for valuation and decision making. Nature Reviews Neuroscience 6, 363.

Vasconcelos, M., Monteiro, T., Aw, J., and Kacelnik, A. (2010). Choice in multialternative environments: a trial-by-trial implementation of the sequential choice model. Behavioural processes 84, 435-439.

Verbruggen, F., and Logan, G.D. (2008). Response inhibition in the stop-signal paradigm. Trends in cognitive sciences 12, 418-424.

Volkow, N.D., Wang, G.-J., Fowler, J.S., Tomasi, D., and Telang, F. (2011). Addiction: beyond dopamine reward circuitry. Proceedings of the National Academy of Sciences 108, 15037-15042.

Wallis, J.D. (2007). Orbitofrontal cortex and its contribution to decision-making. Annu Rev Neurosci 30, 31-56.

Werbos, P. (1974). Beyond regression: New tools for prediction and analysis in the behavioral sciences.

Wang, M.Z., and Hayden, B.Y. (2017). Reactivation of associative structure specific outcome responses during prospective evaluation in reward-based choices. Nature Communications 8, ncomms15821.

Wilson, R.C., Takahashi, Y.K., Schoenbaum, G., and Niv, Y. (2014). Orbitofrontal cortex as a cognitive map of task space. Neuron 81, 267-279.

Xie, Y., Nie, C., and Yang, T. (2018). Covert shift of attention modulates the value encoding in the orbitofrontal cortex. eLife 7, e31507

Yoo, S.B.M., Sleezer, B.J., and Hayden, B.Y. (2018). Robust Encoding of Spatial Information in Orbitofrontal Cortex and Striatum. Journal of cognitive neuroscience, 1-16. 